\title{
Parental Conflicting Role Mediates Regulation of The Chromatin Structure in The Mouse Zygote
}

Masatoshi Ooga ( $\nabla$ mohga@yamanashi.ac.jp)

University of Yamanashi

Rei Inoue

University of Yamanashi

Sayaka Wakayama

University of Yamanashi

Satoshi Kamimura

University of Yamanashi

Teruhiko Wakayama

University of Yamanashi https://orcid.org/0000-0002-6151-2603

\section{Article}

Keywords: zygotes, parental asymmetry, zygotic relaxed chromatin structure, spermiogenesis, spermatozoa, round spermatid, chromatin compaction, chromatin relaxation, intracytoplasmic sperm injection, round spermatid injection

Posted Date: August 26th, 2021

DOl: https://doi.org/10.21203/rs.3.rs-806059/v1

License: (c) (1) This work is licensed under a Creative Commons Attribution 4.0 International License. Read Full License

Version of Record: A version of this preprint was published at Communications Biology on July 14th, 2022. See the published version at https://doi.org/10.1038/s42003-022-03623-2. 
4 Masatoshi Ooga ${ }^{1,2,4}$, Rei Inoue ${ }^{2}$, Sayaka Wakayama ${ }^{1}$, Satoshi Kamimura ${ }^{1,3}$ and 5 Teruhiko Wakayama ${ }^{1}$

6

$7{ }^{1}$ Advanced Biotechnology Center, University of Yamanashi, Kofu-shi, Yamanashi 4008 8510, Japan

$9{ }^{2}$ Faculty of Life and Environmental Science, University of Yamanashi, Kofu-shi, 10 Yamanashi 400-8510, Japan

$11{ }^{3}$ Department of Basic Medical Sciences for Radiation Damages, National Institute of 12 Radiological Sciences, National Institutes for Quantum and Radiological Science and 13 Technology, Chiba 263-8555, Japan.

$14 \quad{ }^{4}$ Corresponding author: mohga@yamanashi.ac.jp 15 16 intracytoplasmic sperm injection, round spermatid injection

\section{Keywords}

zygotes, parental asymmetry, zygotic relaxed chromatin structure, spermiogenesis, spermatozoa, round spermatid, chromatin compaction, chromatin relaxation, 
Abstract

Parental pronuclei (PN) are asymmetrical in several points but the underlying

24 mechanism for this is still unclear. Recently, a theory has been become broadly accepted

25 that sperm are more than mere vehicles to carry the paternal haploid genome into oocytes.

26 Here, in order to reveal the formation mechanisms for parental asymmetrically relaxed

27 chromatin structure in zygotes, we investigated histone mobility in parthenogenetic-,

28 androgenic-, ROSI-, ELSI-, tICSI-, and ICSI-zygotes with several numbers of PNs with

29 the use of zygotic fluorescence recovery after photobleaching, a method previous

30 established by our group. The results showed that sperm played a role to cause chromatin

31 compaction in both parental PNs. Interestingly, during spermiogenesis, male germ cells

32 acquired this ability and its resistance. On the other hand, oocytes harbored chromatin

33 relaxation ability. Furthermore, the chromatin relaxation factor was competed for

34 between PNs. Thus, these results indicated that the parental asymmetrically relaxed

35 chromatin structure was established as a result of a competition between the PNs for the

36 chromatin relaxation factor that opposed the chromatin compaction effect by sperm.

37 Together, it was suggested that parental germ cells cooperated for their just arisen

38 newborn zygotes by playing a distinct role in the regulation of chromatin structure. 
Introduction

During fertilization, parental pronuclei (PN) are formed from the genomes of the sperm and oocyte. Although co-existing in the cytoplasm of the zygote, the PNs are separated before the first mitotic cell cycle and then the two haplotypes fuse to form the new individual genome. There are many differential points in epigenetic factors $(\mathrm{e}, \mathrm{g}$ histone modifications, histone variants and chromatin relaxation) between PNs before fusion. However, the mechanisms underlying parental asymmetry remain unclear and even less is known about how it is controlled by the interactions between the parental PNs.

The results of our previous study revealed that the chromatin of the male PN derived from the sperm (sp-mPN) was comparatively more relaxed during the preimplantation embryonic stages (Ooga et al., 2016). Interestingly, the chromatin of the female PN (fPN) is significantly less relaxed than that of the sp-mPN. Thus, the relaxation of the zygotic parental chromatin structure is asymmetrical in regard to size $\left(0^{\top}>\uparrow\right)$ (Adenot et al., 1997), transcriptional regulation and activity $\left(O^{\prime}>\right.$ ○ ) (Aoki et al., 1997), epigenetic active and repressive of histone markers $\left(\sigma^{7}<\right.$ $<$ ) (Burton et al., 2008) and the amounts of reprogramming factors $\left(\sigma^{\prime \prime}>\right.$ Q ) (Liu et al., 2014). Round spermatid are haploid precursor cells present during the spermatogenetic stage soon after meiosis. Importantly, round spermatid injection (ROSI) and delay of intracytoplasmic sperm injection (ICSI) can result in improper PN formation and subsequent developmental failure during the preimplantation stage (Kishigami et al., 2004b), suggesting that the

61 importance of parental asymmetry. However, the mechanism underlying parental asymmetry has not yet been elucidated. In addition, it is unknown whether relaxation of 
the asymmetrical parental chromatin is due to an acquisition of greater extent of relaxation of the chromatin of the sp-mPN or compaction of the fPN. Furthermore, the molecules involved in relaxation of the asymmetrical parental chromatin have not yet been identified.

It has recently become clear that the spermatozoon also plays a role in the regulation of the embryonic chromatin structure. For example, sperm carry epigenetic factors responsible for the highly complex organization of the genome (Brykczynska et al., 2010; Hammoud et al., 2009; Paradowska et al., 2012) and DNA/histone modification and RNA in the zygote (Yamaguchi et al., 2018; Sharma et al., 2016), which were thought to be involved in the regulation of the establishment of the zygotic chromatin structure and contribute to the control of embryonic development (Trigg et al., 2019; Teperek et al., 2016; Brykczynska et al., 2010; Chen et al., 2021; Sharma et al., 2016). Although the contribution of spermatozoa to the establishment of the zygotic chromatin structure has been widely investigated, it remains unknown not only whether the molecular properties of sperm are involved in establishing the extremely relaxed structure of the sp-mPN chromatin and but also whether these factors are actively involved in establishing the asymmetric relaxation of the parental chromatin after fertilization.

Therefore, the aim of the present study was to investigate the mechanisms underlying the asymmetric relaxation of the parental chromatin. The results of this study revealed that sp-mPN harbored the ability to further compact the chromatin of the fPN, resulting in asymmetric relaxation of the parental chromatin structures. In addition to the ability of the sperm to further compact the chromatin, our results indicated that the parental PNs compete to relax the chromatin. Thus, the chromatin structure of the zygote is regulated by the chromatin compaction effect derived from the sperm and the chromatin 
87 relaxation effect derived from oocytes in opposition to the sperm-derived compaction

88 effect. Hence, the asymmetrical chromatin relaxation of the zygotic is established by 89 interactions between the parental germ cells.

90

91

92 
Results

114 dependent chromatin compaction were also activated in the sp-mPN itself, 1PN-ICSI

\section{Sperm causes compaction of both parental chromatin structures}

We previously reported that asymmetric relaxation of the parental chromatin was established in the late zygotic stage of the embryo at 10-12 hours post insemination (hpi) (Ooga et al., 2016). First, we confirmed the reproducibility of asymmetric relaxation of the parental chromatin $\left(\sigma^{\top}>\right.$ $>$ ) in zygotes obtained by in vitro fertilization (IVF) (Fig. S1) and ICSI. In addition, the mechanisms causing the parental asymmetric pattern were investigated by determining whether sp-mPN acquired the highly relaxed or fPN obtained the compacted chromatin structure. To this end, the dynamics of chromatin relaxation during the early to mid-zygotic stages were examined with the use of parthenogenetically activated- and ICSI-derived zygotes. Although the chromatin of the fPN was gradually compacted along with the development of the zygote, there was no significant change in the chromatin of the sp-mPN (Fig. 1A and B). Importantly, in the presence of sperm/sp$\mathrm{mPN}$, the chromatin of the fPN was further compacted. As a result, the parental asymmetric pattern $\left(0^{\prime}>\right.$ Q $)$ was established by 8 hpi. The dependency of fPN compaction on sp-mPN was confirmed by enucleation of the sp-mPN followed by immuno-staining of histone 3 lysine 9 trimethylation $(\mathrm{H} 3 \mathrm{~K} 9 \mathrm{me} 3)$ as a marker of the fPN (Fig. 1C and S2). Collectively, these findings suggest that the asymmetrical structure of the parental chromatin was established via acquisition of the compacted chromatin structure of the fPN by a mechanism dependent on the sperm/sp-mPN.

Next, in order to determine whether the mechanisms underlying sp-mPNwere constructed by ICSI with enucleated MII oocytes (Fig. 1D). The chromatin of a 
Fig. 1
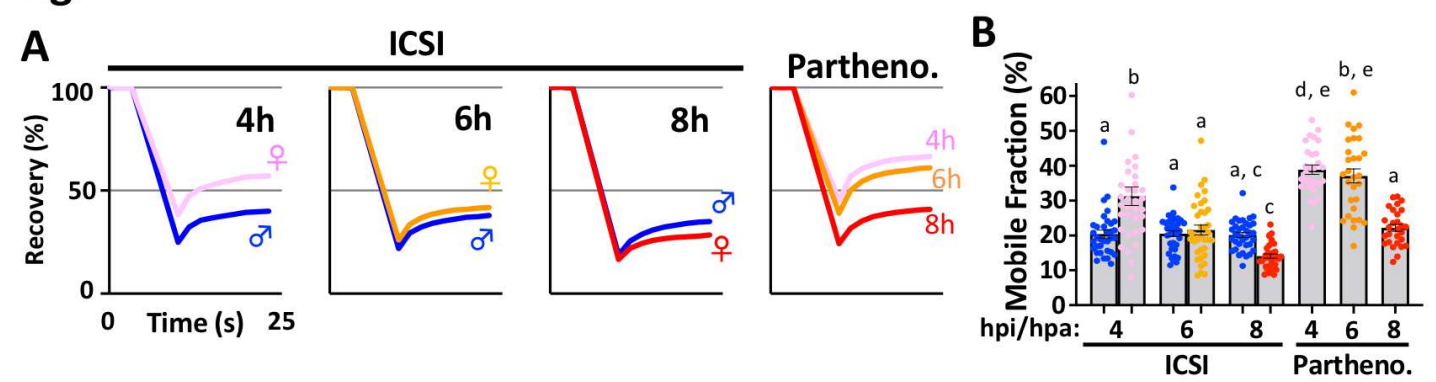

C

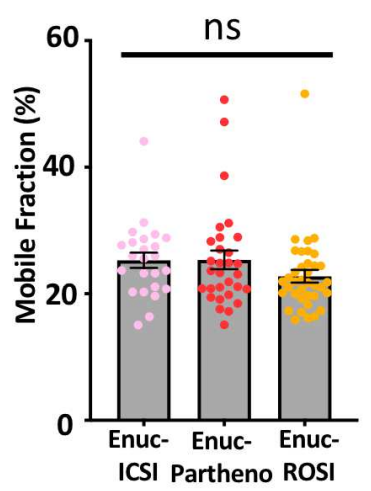

D

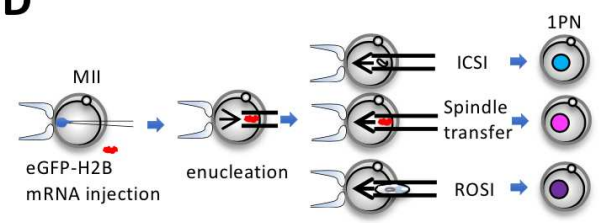

E
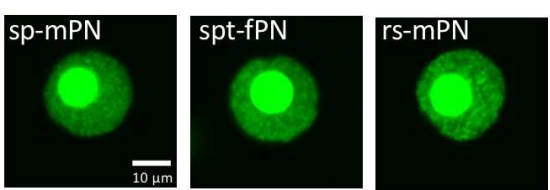

$\mathbf{F}$

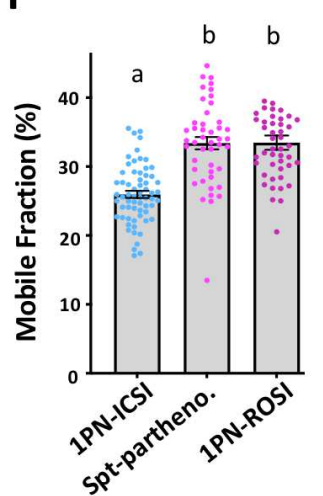

G

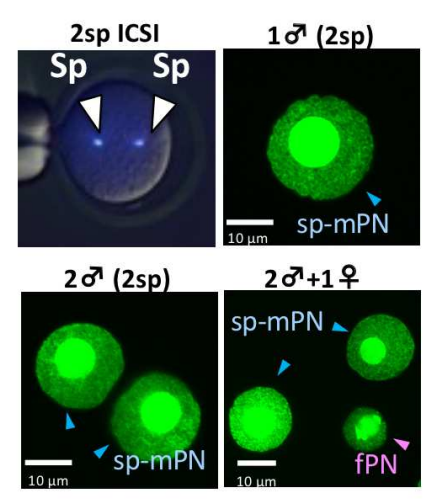

H

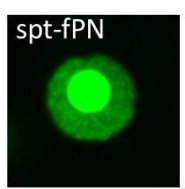

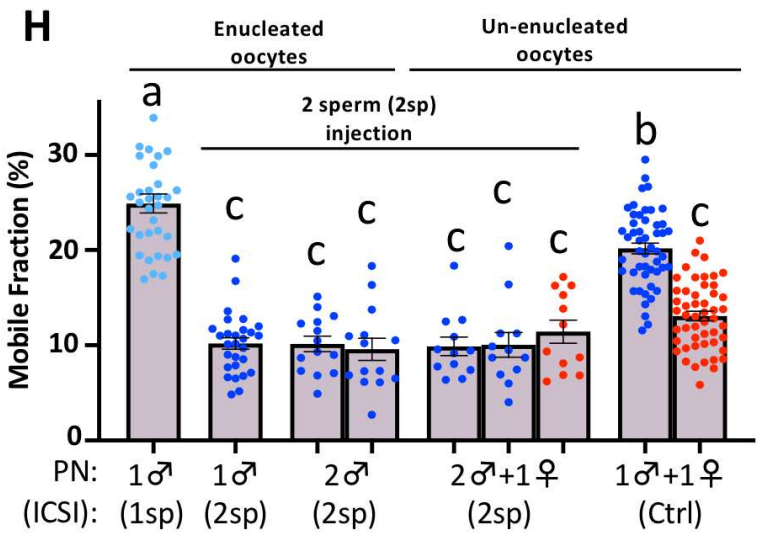

$$
\begin{array}{llll}
\text { (ICSI): (1sp) (2sp) (2sp) (2sp) } & &
\end{array}
$$
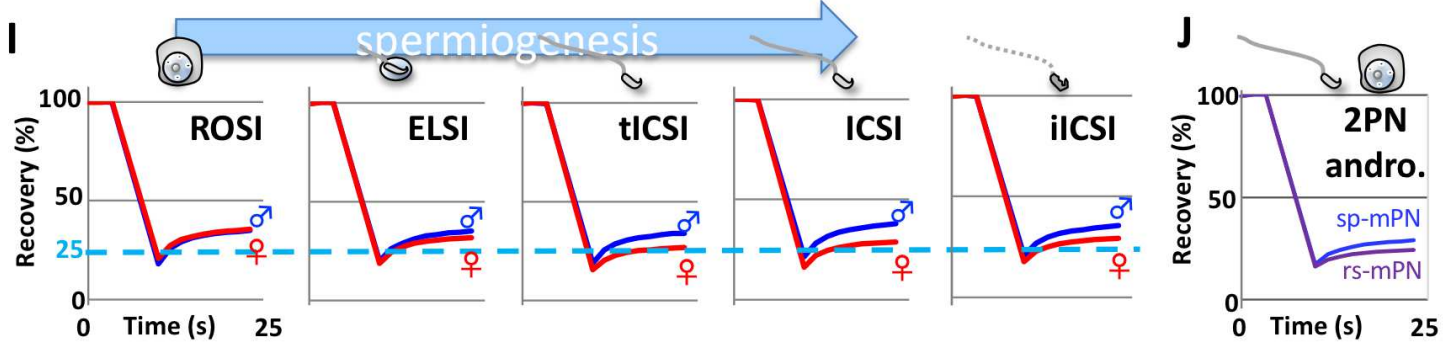

117 Fig. 1. Sperm causes compaction of both parental chromatin structures

118 (A and B) Dynamics of chromatin relaxation during the early and mid-stages of ICSI

119 and parthenogenetically activated zygotes. zFRAP analysis was performed at 4, 6 , and 8

120 hpi or hpa. A recovery curve indicating the average fluorescence recovery rate is shown 
121 (A). The average mobile fraction (MF) is shown as a gray bar (B). Single dots indicate

122 the MF score of each male and female pronuclei (mPN, fPN), respectively. Blue, mPN;

123 pink, fPN-4h; orange, fPN-6h; and red, fPN-8h. For parthenogenetic (partheno)-zygotes

124 with 2PN, the average MF score is shown. Error bar indicates the standard error (SE). (C)

$125 \mathrm{mPN}$ was enucleated at $4 \mathrm{hpi} / \mathrm{hpa}$ from ICSI- and ROSI-zygotes. Two PN partheno-

126 zygotes were prepared as controls. The remaining fPN was subjected to zFRAP analysis

127 at $8 \mathrm{hpi} / \mathrm{hpa}$. (D) Illustration of the preparation of 1PN-zygotes (E) Fluorescence images

128 of each single PN: sperm-derived mPN (sp-mPN), spindle transfer-derived fPN (spt-fPN),

129 and round spermatid-derived mPN (rs-mPN) (F) Average MF scores of 1PN-ICSI, -ROSI,

130 and spt-partheno. (G) Two sperm were injected into enucleated MII oocytes (upper-left).

131 Sperm (sp) were stained with Hoechst 33342. Fluorescence images of PNs: "1 o" (2sp)"

132 and " $20^{\text {" }}(2 \mathrm{sp})$ " indicate the one and two male PN-zygotes injected with two sperm (2sp),

133 respectively (upper right and lower left). Two sperm injected into un-enucleated MII

134 oocytes $\left(20^{7}+1\right.$; ; lower right). (H) Average MF scores of the zygotes as shown in $(\mathbf{G})$.

135 As a control, one sperm was injected into enucleated MII- (same as 1PN-ICSI in Fig. 1

$136 \mathrm{~F} ;$ " “ $1 \mathrm{O}^{\prime}(1 \mathrm{sp})$ ”)) and normal ICSI-zygotes $\left(1 \mathrm{O}^{\prime}+1\right.$ + ). Blue and red dots indicated mPN

137 and fPN, respectively. (I) Recovery curve of ROSI-, ELSI-, tICSI-, ICSI-, and iICSI-

138 zygotes. (J) Recovery curve of androgenic zygotes prepared by co-injetion of sperm and 139 round spermatid. 
140 single sp-mPN was more compact than a single fPN of parthenogenetic zygotes, although

141 both appeared similar, suggesting that the chromatin compaction mechanism of sp-mPN

142 worked on its own (Fig. 1E, F and S3). Further confirmation by ICSI was conducted with

143 the use of enucleated MII oocytes fertilized with two sperm (2sp) (i.e., "10" (2sp)"and

144 "20" (2sp)" in Fig. 1G, H and S4), which showed that the additional sperm resulted in

145 further chromatin compaction in each sp-mPN, as the chromatin of zygotes formed by

146 ICSI with two sperm and an enucleated MII oocyte was more compact than that of the

147 zygotes formed by one sperm ("10" (1sp)," light blue in Fig. 1F and H). In zygotes

148 formed by ICSI with two sp-mPNs and an "un-enucleated" oocyte (" 20 " 1 \%"), two sp-

149 mPNs were comparable to one fPN and disruption of the parental asymmetric pattern

$150\left(\sigma^{\prime} \fallingdotseq\right.$ ? ). Importantly, there was no significant difference between the fPNs of $20^{7}+1$ 우

151 and that of $10^{7}+1 \%$ (ctrl), indicating that the chromatin of the fPNs was already

152 compacted to almost the possible limit even in the presence of only one sperm. On the

153 other hand, to compact the chromatin of sp-mPN to this level, at least two sperm were

154 needed. Thus, the sp-mPN exhibited innate resistance to chromatin compaction. Together,

155 these results suggest that although the chromatin compaction effect works on the

156 chromatin of both parental PNs, asymmetric relaxation of the parental chromatin was

157 established due to differences in sensitivity to this effect. The zygotes harboring two fPNs

158 with a single sp-mPN ("1 $\mathbf{1}+\mathbf{2}$ \%”) still exhibited the parental asymmetric pattern (Fig.

159 S5). Thus, the additional chromatin from the fPN failed to disrupt the parental asymmetric 160 pattern.

161 To examine where the ability for the chromatin compaction in both

162 parental PNs was acquired among the stages during spermiogenesis, zygotic 
163 fluorescence recovery after photobleaching (zFRAP) analysis of zygotes fertilized by 164 various micro-insemination methods (ROSI, ELSI, tICSI, and ICSI) was performed at 8 165 hpi or hours post micro-activation (hpa). All male PNs of zygotes obtained by ROSI and 166 ICSI exhibited similar levels of chromatin relaxation (Fig. 1I and Fig. S6A). By contrast, 167 the extent of chromatin relaxation of the fPNs of these zygotes was decreased along with 168 the maturity of the male germ cells. Thus, the maturity of spermatogenic cells is correlated 169 with the asymmetric structure of the parental chromatin. The asymmetric structure of the 170 parental chromatin in zygotes obtained by ICSI with the use of inactivated sperm (iICSI171 zygotes) was only slightly decreased as compared to that of the ICSI-zygotes (Fig. 1I and

172 Fig. S6B), indicating that asymmetric relaxation of the parental chromatin acquired 173 during spermiogenesis could not be explained by the activation capacity of the oocyte.

174 Taken together, these results suggest that sperm has the ability to compact the chromatin 175 of both parental PNs and resistance to compaction leads to parental asymmetrically 176 relaxed chromatin structure during spermiogenesis. Correct discrimination of parental 177 chromatin in ROSI-zygotes was confirmed by zFRAP analysis with paternal PNs from 178 enucleated zygotes followed by immunocytochemical analysis of H3K9me3 as a marker 179 of the fPNs (Fig. S7). The inability of round spermatid to compact chromatin was 180 confirmed by 1 PN-ROSI, which showed a comparable level of chromatin relaxation of 181 1PN-parthenogenetic zygotes (Fig. 1F, purple). In addition, 2PN androgenic zygotes 182 formed by sp-mPN, with comparatively greater chromatin relaxation, and round 183 spermatid-derived from the male PN (rs-mPN), with relatively less chromatin relaxation

184 (Fig. 1J and S8), indicated that the round spermatid had not yet acquired resistance to 185 chromatin compaction. Furthermore, in the presence of sp-mPN, the chromatin of the rs$186 \mathrm{mPN}$ was condensed to the same level as that of the fPN (Fig. S9). These findings were 
consistent with the disruption of the asymmetric structure of the parental chromatin in zygotes obtained by ROSI.

\section{Parental PNs compete for chromatin relaxation factors}

As shown in Fig. 1F and $\mathbf{H}$, the chromatin of 1PN-zygotes and even 1PN ICSIzygotes, was extremely relaxed as compared to that of ICSI-zygotes with two parental PNs $\left(10^{7}(1 \mathrm{sp})\right.$ vs. $10^{7}+1$ ( $\left.(1 \mathrm{sp})\right)$. The results of our previous study revealed that oocytes harbored highly loosened chromatin structures and chromodomain helicase DNA binding protein 9 (CHD9), which regulates chromatin remodeling, participated in the regulation of the chromatin relaxation (Ooga et al., 2018b). Furthermore, in another previous study, the transferred somatic cell nuclei into enucleated oocytes acquired a relaxed chromatin structure, indicating the presence of factors promoting chromatin relaxation in oocytes and zygotes (Ooga et al., 2016). These findings prompted the hypothesis that the concentration of factors promoting chromatin relaxation into a single PN led to the extremely relaxed chromatin structure. At the same time, such factors were distributed to the parental PNs. To examine this possibility, parthenogenetically activated oocytes were constructed with various numbers of fPNs (1, 2, and 4 fPNs; Fig. 2A), which enabled exclusion of the sp-mPN-derived chromatin compaction effect. As expected, the extent of chromatin relaxation decreased along with the increase in the number of PNs and the fPNs in the same zygotes showed similar level of chromatin relaxation (Fig. 2B and S10). These results suggest that the chromatin relaxation factors are present in the zygotes and at least, in parthenogenetic zygotes, the fPNs competed for these factors.

If the parental PNs compete for factors that promote chromatin relaxation, the lack of one parental PN should cause excess chromatin relaxation in another. Therefore, 
Fig. 2

A

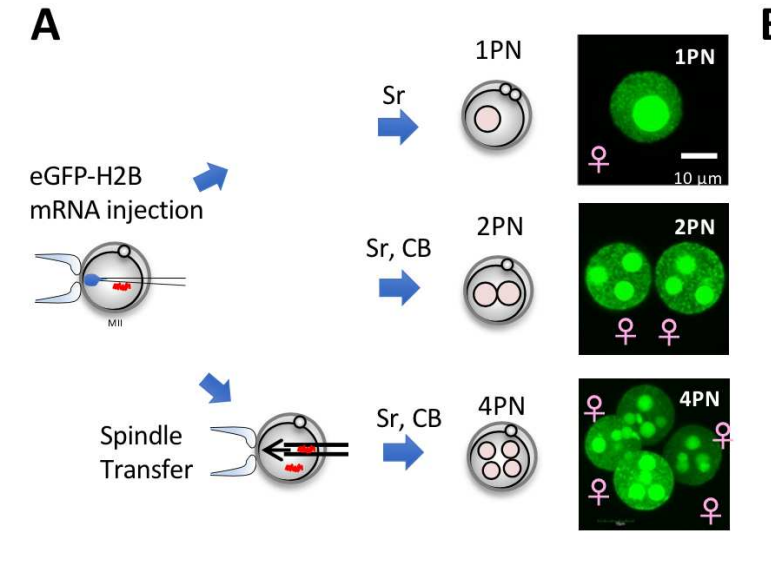

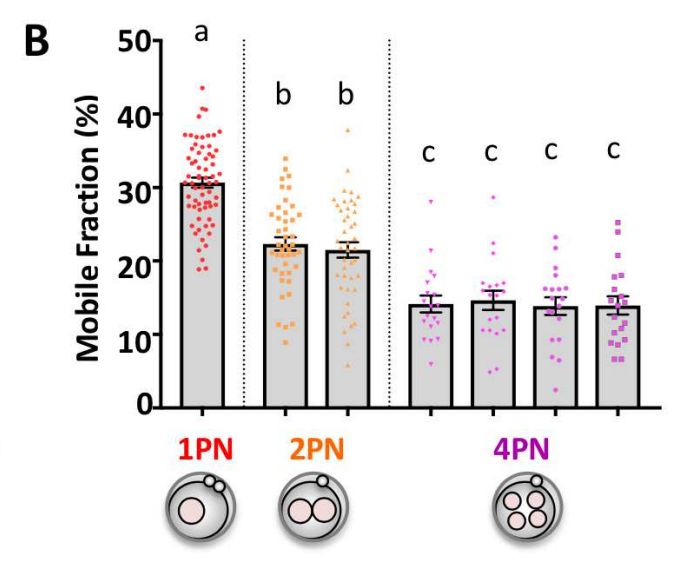

C
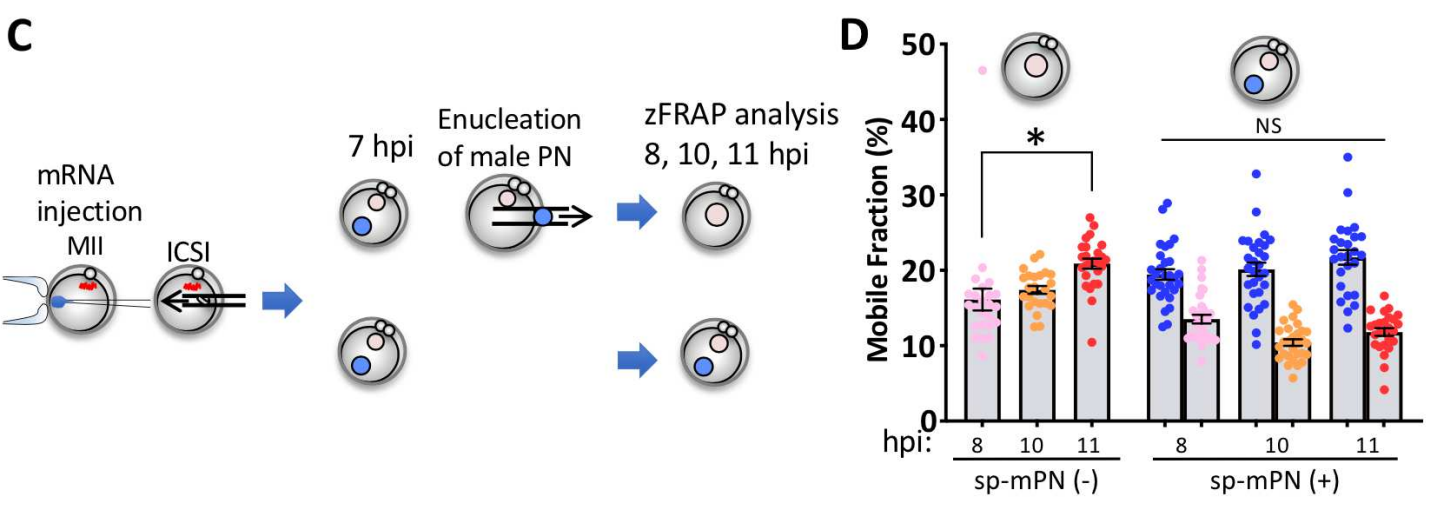

$\mathbf{E}$

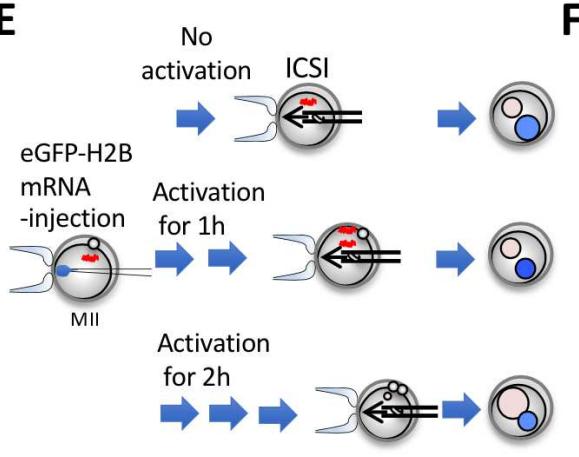

$\mathbf{F}$

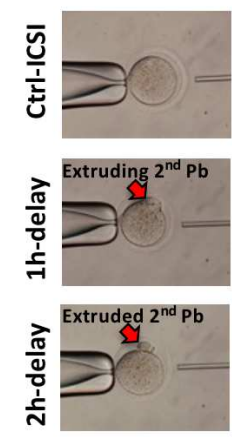

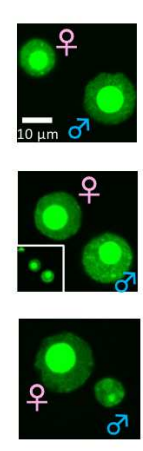

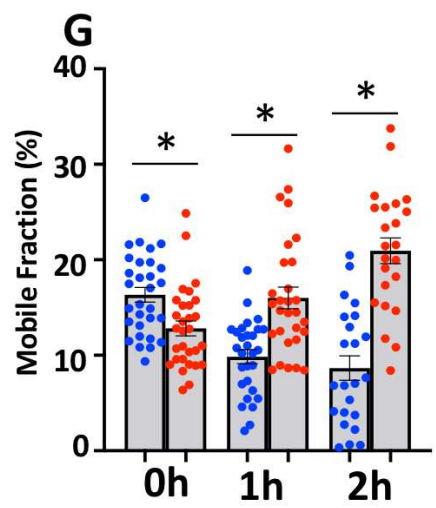

212 Fig. 2. Parental PNs compete for chromatin relaxation factors

213 (A) Illustration of the preparation of 1, 2, and 4PN partheno-zygotes and fluorescence

214 images of the fPNs (B) Average MF scores of the partheno-zygotes prepared as shown in

215 (A). (C) Illustration of the preparation of sp-mPN-enucleated partheno-zygotes. (D)

216 Average MF scores of the zygotes prepared as shown in $(\mathbf{C})$. Blue, mPN; pink, fPN-8h;

217 orange, fPN-10h; and red, fPN-11h). Asterisks indicate significant differences. (E) 
218 Illustration of the preparation of delay ICSI-zygotes. "Sr" indicates strontium. (F) Control 219 and delay ICSI are shown. The second polar body is indicated with a red arrow. 220 Fluorescence images of the $\mathrm{mPN}$ and $\mathrm{fPN}$. The inset shows lower magnification images 221 of the second polar body near the fPN. (G) Average MF scores of the delay ICSI-zygotes 222 prepared as shown in $(\mathbf{E}, \mathbf{F})$. 
the potential of excess chromatin relaxation in fPN was investigated with the use of

225 enucleation of sp-mPNs. In sp-mPN-enucleated-zygotes, chromatin relaxation of the fPN 226 gradually increased along with the progression of zygotic development (Fig. 2C and D).

227 In contrast, zFRAP analysis of ICSI-zygotes at 8, 10, and 11 hpi showed that the extent 228 of chromatin relaxation was maintained in both parental PNs. Thus, the parental PNs competed for the chromatin relaxing factors. Next, the effect of delayed PN formation (Kishigami et al., 2004b) on parental asymmetry was investigated. To this end, delayedICSI zygotes were constructed and then analyzed by zFRAP (Fig. 2E). Observation of

232 PN formation of delayed-ICSI zygotes revealed the reversal of PN size, larger or smaller, 233 between parental PNs (Fig. 2F). zFRAP analysis revealed that asymmetric relaxation of the parental chromatin was compromised along with an increased delay time of ICSI (Fig. 2G and S11). Particularly, almost all of the $2 \mathrm{~h}$-delayed-ICSI zygotes showed a reversed parental asymmetric pattern $\left(\sigma^{7}<\uparrow\right)$. A delay of only $1 \mathrm{~h}$ resulted in a considerable change in PN size and the chromatin of the fPN was more relaxed than that of the spmPN. Collectively, these results indicate that parental PNs compete for chromatin relaxing factors and the state of the zygotic chromatin is regulated by an antagonistic

240 balance between the chromatin compaction effects derived from the sperm and the relaxation effect from the oocyte. Furthermore, it is possible that the sp-mPN might have obtained more such relaxation factors than the fPN, resulting in self resistance to the chromatin compaction effect.

246 Finally, three types of RNA polymerase inhibitors (i.e., actinomycin D ("Act 
248 IIIi) were employed to determine whether chromatin relaxation factors are produced by 249 zygotic transcription. Ama slightly, but not significantly, increased the extent of 250 chromatin relaxation (Fig. S12), indicating that the chromatin relaxation factors were not 251 derived from Pol II-transcribed mRNA. However, both Act D (Fig. 3A and B) and Pol 252 1lli (Fig. 3C and D) caused significant compaction of the chromatin in the only sp-mPN. 253 Thus, relaxation of the chromatin of the sp-mPN was more sensitive to these inhibitors 254 than that of the fPN, suggesting that more chromatin relaxation factors were utilized the 255 in sp-mPN. Furthermore, since Pol I and III produce RNA that is involved in translation, 256 it is possible that chromatin relaxation factors are proteins, such as H1foo (Funaya et al., 257 2018), presumably also supplied by zygotic translation of maternally stored mRNA. 258 259 
Fig. 3

A

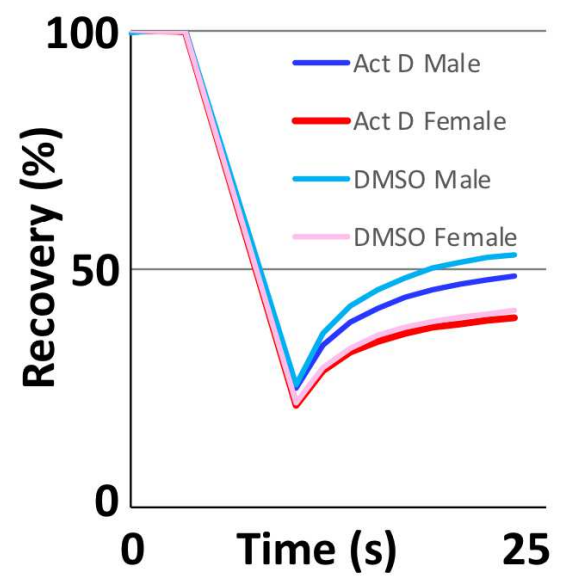

C

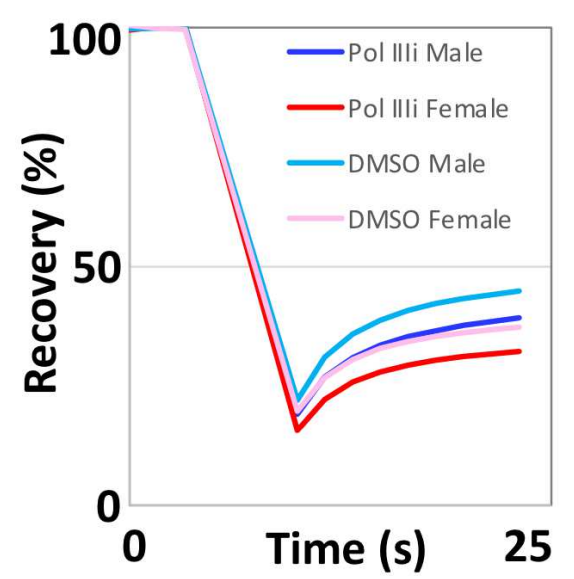

E

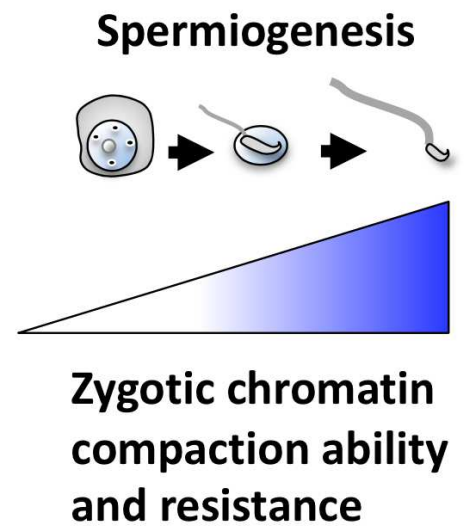

B

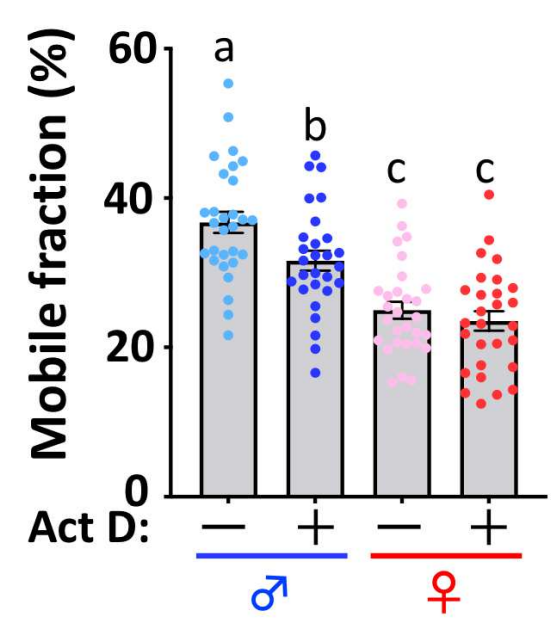

D

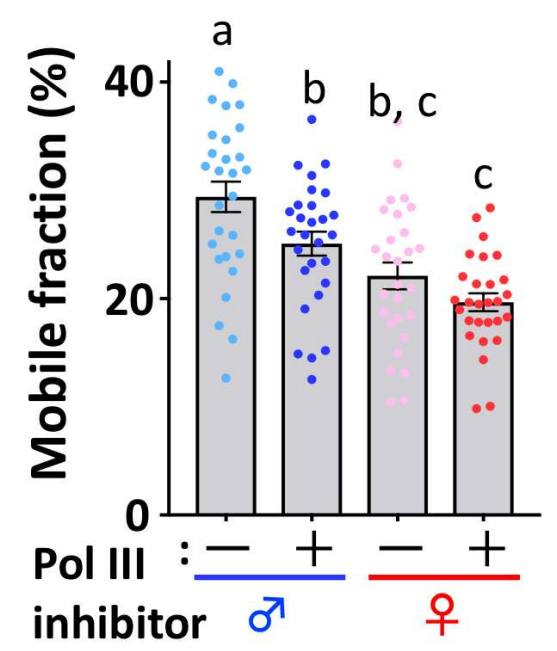

F

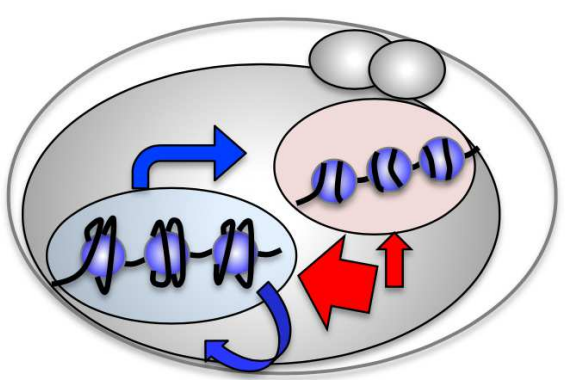

Chromatin compaction (sperm)
Chromatin relaxation (oocytes)

261 Fig. 3. More chromatin relaxer was utilized in sp-mPN than fPN 
262 (A) Recovery curve of IVF zygotes treated with $0.1 \mu \mathrm{g} / \mathrm{ml}$ Act D. Control zygotes were 263 treated with 1\% dimethyl sulfoxide (DMSO). (B) Average MF scores of IVF zygotes 264 treated with Act D and DMSO. (C) Recovery curve of IVF-zygotes treated with $20 \mu \mathrm{M}$ 265 Pol IIIi. Control zygotes were treated with 0.1\% DMSO. (D) Average MF scores of IVF 266 zygotes treated with Pol IIIi and 0.1\% DMSO. (E) Schematic illustration indicating that 267 male germ cells acquire the ability to compact chromatin and resistance during 268 spermiogenesis. (F) In the zygotes, the sperm-derived chromatin compaction effect and 269 oocyte-derived chromatin relaxation factors are antagonistic. Probably, more chromatin 270 relaxation factors caused a more relaxed state and conferred resistance to chromatin 271 compaction in the sp-mPN.

272 
Discussion

In this study, the mechanisms underlying asymmetric relaxation of the parental

275 chromatin were investigated, which revealed that sperm have the ability to compact the

276 chromatin of both parental PNs (Fig. 1A-H). Interestingly, the abilities to promote and

277 resist chromatin compaction are acquired during spermiogenesis (Fig. 1I, J and 3E). In

278 addition to the ability to compact chromatin, zygotes also harbor factors that promote

279 chromatin relaxation, which the parental PNs compete for (Fig. 2A-G) and are

280 presumably dependent on zygotic translation of maternally pooled mRNA (Fig. 3A-D).

281 Thus, asymmetrically relaxed chromatin of the zygote is established via the mature male

282 and female germ cells (Fig. 3F), and is possibly determined by a balance between them.

283 Hereafter, this power balance is referred to as "parental epigenetic competition."

In 1PN-ICSI zygotes, the sp-mPN demonstrated less chromatin relaxation than

that of the 1PN-ROSI and spindle-transferred haploid-parthenogenetically activated oocytes (Fig. 1F and S3). This result indicated that sperm actively condense the

287 chromatin structure. In Fig. 1I and S6, although $\mathrm{NaOH}$-treated inactivated sperm lost the ability to activate the oocyte, the ability to compact the chromatin was retained, indicating that the unidentified sperm-derived chromatin condensing factors are not associated with the sperm surface. A recent broadly accepted theory states that sperm are more than mere

291 vehicles to carry the paternal haploid genome into the oocyte. Indeed, sperm carried huge

292 kinds of RNA into oocytes at fertilization. several studies showed during epididymal 293 transit from testis to cauda epididymis, sperm obtained small RNA payload (Sharma 2019; Trigg et al., 2019). Since tICSI-zygotes, which have no such RNA payload, exhibit asymmetric relaxation of the parental chromatin, the RNA payload of the mature sperm might not be involved in compaction of the zygotic chromatin. However, the possibility 
297 that sperm RNA is involved in compaction of the chromatin compaction must still be 298 considered. Reportedly, sperm RNA is deeply embedded in the sperm head (Yan et al., 299 2008). Such RNA is not easy to extract and $\mathrm{NaOH}$ treatment did not completely dissolve 300 the sperm head, indicating that the RNA was not eliminated (Schuster et al., 2016). In 301 addition to the embedded RNA, proteins are possibly the responsible factors. Following 302 the initiation of spermiogenesis, during which there is no transcription, specific stored 303 RNAs were translated to proteins (Rathke et al., 2014). Since our results indicated that 304 the ability of sperm to compact chromatin is acquired after the initiation of 305 spermiogenesis, it is also possible that newly synthesized proteins at this phase are 306 responsible for chromatin compaction. To understand the importance and mechanisms of 307 parental epigenetic competition, further studies are needed to test this hypothesis and to identify the RNA and/or protein molecules responsible for zygotic chromatin compaction. It is possible that there are differences in the dynamics of PN formation

310 involved in the regulation of parental epigenetic competition. The establishment of the 311 chromatin structure of the sp-mPN is very distinct from that of the fPN. Within $1 \mathrm{~h}$ after

312 fertilization, maternally pooled histone proteins are rapidly incorporated into the sp-mPN,

313 resulting in the sperm head becoming decondensed and expanded ${ }^{21}$. On the other hand,

314 at this phase, maternal genetic materials still form completely condensed meiotic 315 chromosomes, which are located in the cytoplasm or the going to be extruded second 316 polar body (Fig. 2F; Ooga et al., 2008). Also, it is widely thought that the transcription 317 factors and chromatin remodeling factors dissociate from the condensed chromosomes 318 and are re-recruited to the re-organized chromatin structure after chromosome 319 segregation ${ }^{23}$. Therefore, it is likely that maternal factors were first taken up by the sp$320 \mathrm{mPN}$ and then later by the fPN. In $1 \mathrm{~h}$-delayed-ICSI-zygotes, the asymmetric relaxation 
321 of the parental chromatin structure was reversed (Fig. 2G and S11). This experiment was

322 designed to collapse the competition for the maternally supplied factors from the ooplasm 323 and resulted in reversal of the parental asymmetry. Thus, it is possible that more 324 maternally pooled or newly produced zygotic factors were incorporated into the sp-mPN 325 than the fPN in normally fertilized zygotes. Indeed, more reprogramming factors that 326 confer totipotency to the somatic cell nuclei are reportedly incorporated into the sp-mPN than the fPN (Liu et al., 2014). Accordingly, it is plausible that more maternally supplied chromatin relaxation factors could be incorporated into the sp-mPN than the fPN, resulting in asymmetric relaxation of the parental chromatin. and S3). Moreover, when compared, in 1PN-zygotes, rs-mPNs and fPNs, which are derived from transferred meiotic spindles, exhibited the same level of chromatin relaxation. However, there was significant asymmetric relaxation of the parental

334 chromatin in ROSI-zygotes (Fig. 1I, S6). Our ROSI-zygote production strategy employed 335 a "post-activation protocol" (Kishigami et al., 2004a) to improve the rate of 2PN 336 formation (Kishigami et al., 2004b). In this protocol, the oocytes injected with round 337 spermatids were activated within 30 min after ROSI. As a result, the round spermatid 338 genome was able to avoid premature chromatin condensation followed by extrusion of 339 the pseudo polar body. Thus, chromosome condensation and incorporation of maternal 340 factor did not seem to be equal between the rs-mPN and fPN, suggesting the possibility 341 that the rs-mPN harbored more chromatin relaxation factors than the fPN (Fig. S13).

342 Collectively, these findings suggest that it is probable that the differences in dynamics 343 during PN formation contribute to the parental epigenetic competition. 
345 compaction effects in both parental PNs. This finding raises the question of the biological

346 significance of zygotic chromatin compaction by sperm. Bui et al. reported that sperm

347 have the ability to regulate transcriptional activity (Bui et al., 2011). Thus, sperm play an

348 important role in the regulation of zygotic genome activation (ZGA). Chromatin 349 compaction by sperm might be involved in regulation of ZGA. It was thought that 350 promiscuous transcription occurs during minor ZGA and correlates with extensive 351 chromatin relaxation by FRAP (Abe et al., 2018). Our results demonstrated that sperm352 derived chromatin compaction factors condense the paternal chromatin structure in the 353 sp-mPN and then the extent of chromatin relaxation becomes comparable to that of the rs-mPN, indicating that in the absence of sperm-derived chromatin condensing factors, the paternal chromatin structure derived from the sperm should be extremely relaxed.

356 Then, it is possible that such an extreme chromatin structure will cause abnormalities to 357 the transcriptome during ZGA. To assess this possibility, the chromatin compaction 358 factors must be identified with the use of a knockdown/knockout experimental system, which was not possible in the current study. We also observed abnormal chromatin relaxation in the ROSI-zygotes.

361 Therefore, it would be beneficial to analyze the transcriptome of ROSI-derived embryos 362 to understand biological role of chromatin relaxation. The results of this study confirmed 363 that that sperm actively participate in the regulation of asymmetric relaxation of the 364 parental chromatin structure. However, the reason why the chromatin of the mPN is more 365 relaxed than that of the fPN remains unclear. Hence, comparative analysis with RNA-seq 366 of control- and 1 h-delay-ICSI-zygotes is warranted. Nonetheless, further investigations 367 are needed to understand the significance of parental epigenetic competition. 
Animals

Eight to 12-week-old female B6D2F1 $(\mathrm{C} 57 \mathrm{BL} / 6 \times \mathrm{DBA} 2)(\mathrm{n}=102)$ and 1014-week-old male ICR $(\mathrm{n}=42)$ mice (SLC, Shizuoka, Japan) were used as oocyte and spermatozoa donors, respectively. All animal experiments were approved by the Ethics Committee of the University of Yamanashi (reference number: A29-24) and conducted in accordance with Guide for the Care and Use of Laboratory Animals and the ARRIVE guidelines. All mice were housed under specific pathogen-free conditions at a constant temperature of $25^{\circ} \mathrm{C}$, relative humidity of $50 \%$, and a $14 / 10$-h light/dark period with ad libitum access to a commercial diet and distilled water. In this study, body weight was not measured because the body weight of young mice has no effect on embryo quality.

ICSI and ROSI

Obtained cumulus cells and oocyte complexes were treated with hyaluronidase for $10 \mathrm{~min}$ and the denuded oocytes were collected. For ICSI, spermatozoa were obtained

384 from the cauda epididymis and then cultured in human tubal fluid ${ }^{27}$ for capacitation. Prior to cytosolic injection of the denuded oocytes, the sperm tails were eliminated with a Piezo

386 drive micromanipulator (Prime Tech Ltd., Ibaraki, Japan) in CZB-HEPES medium 387 supplemented with 10\% PVP (10\% PVP-CZB-HEPES) (Chatot et al., 1990). The zona 388 pellucida and cytosolic membranes were also disrupted with a Piezo drive micromanipulator. For ROSI, ELSI, and testicular ICSI, the harvested testes were minced with scissors, sieved through a Mini Cell Strainer, and then re-suspended in 10\% PVP-

391 CZB-HEPES. The nucleus of each round spermatid was collected with a narrow pipette 
with a diameter of 7-8 $\mu \mathrm{m}$. The zona pellucida and cytosolic membrane were disrupted

393 in the same manner as for ICSI. The oocytes injected with round spermatid were activated

394 by culturing in $\mathrm{Ca}^{2+}$-free $\mathrm{CZB}$ medium containing $5 \mathrm{mM} \mathrm{SrCl}_{2}$ for $1-2 \mathrm{~h}$. The tails of the

395 testicular sperm were also cut with a Piezo drive micromanipulator as with ICSI.

396

Enucleation and injection of the nuclei of oocytes

containing HEPES-buffered CZB. After $10 \mathrm{~min}$, the nuclei were aspirated with a glass capillary tube (Wakayama et al., 2019). After enucleation, the ooplasm was washed and cultured in CZB until micro-insemination. In some experiments, the aspirated nuclei were injected into enucleated ooplasms or un-enucleated MII oocytes in CB containing HEPES-buffered CZB (Konno et al., 2020).

\section{Enucleation of male PN}

407 (Lawitts et al., 1993) containing CB for $20 \mathrm{~min}$. The zygotes were then transferred into

408 CB containing HEPES-buffered CZB. The larger PN and furthest away from the second 409 polar body was deemed the mPN, which was aspirated from the zygote. The enucleated 410 zygotes were washed and cultured in KSOM.

412 Delay ICSI

413 Collected oocytes were subjected to parthenogenetic activation in $\mathrm{Ca}^{2+}$-free

$414 \mathrm{CZB}$ medium containing $5 \mathrm{mM} \mathrm{SrCl}_{2}$. After $1 \mathrm{~h}$, the activated oocytes with extruding 415 second polar bodies were collected for micro-insemination with capacitated spermatozoa. 
416 At $2 \mathrm{~h}$ after activation, the zygotes with an obvious extruded second polar body were used

417 for micro-insemination.

418

In vitro fertilization

Spermatozoa were obtained from ICR mice. For capacitation, the spermatozoa were cultured for $1 \mathrm{~h}$ before insemination. Cumulus cells and oocyte complexes were obtained from super-ovulated BDF1 female mice by injection of 7.5 IU of equine chorionic gonadotropin (ASKA Pharmaceutical Co., Ltd., Tokyo, Japan) and human chorionic gonadotropin (ASKA Pharmaceutical) at 46-50-h intervals. Cumulus cells and oocyte complexes were inseminated with capacitated sperm in human tubal fluid medium supplemented with bovine serum albumin (BSA; Sigma-Aldrich Corporation, St. Louis, MO, USA) at $3 \mathrm{mg} / \mathrm{ml}$. At $1-2 \mathrm{~h}$ post-insemination, the zygotes were washed and cultured in $\mathrm{KSOM}$ medium under humidified atmosphere of $5 \% \mathrm{CO}_{2} / 95 \%$ air at $38^{\circ} \mathrm{C}$.

The plasmid "pTOPO eGFP-H2B” (Ooga et al., 2016) encoding enhanced green fluorescent protein (eGFP)-fused histone H2B was linearized by Not1 overnight. Afterward, the plasmid was purified with phenol/chloroform and then precipitated with 434 ethanol. Purified DNA was dissolved in nuclease-free water as template DNA for 435 subsequent in vitro transcription with using mMESSAGE MACHINE sp6 kit (Themo 436 Fisher Scientific, MA, USA). Synthesized mRNA was then processed with a poly A 437 tailing kit (Themo Fisher Scientific). The mRNA with a poly A tail was purified and 438 precipitated with lithium chloride precipitation solution, dissolved, and stored at 500 $439 \mathrm{ng} / \mu \mathrm{l}$ and $-80^{\circ} \mathrm{C}$ until use. 

mRNA encoding eGFP-H2B (250 ng/ $\mu \mathrm{l})$ was prepared as shown above and

443 injected into the cytoplasm of unfertilized MII oocytes or zygotes at $1-2 \mathrm{~h}$ after 444 insemination. mRNA-injected MII oocytes were then micro-inseminated with a round spermatid, elongated spermatid, or spermatozoa. At $8 \mathrm{~h}$ post-insemination or -activation, the zygotes were collected for zFRAP analysis, which was performed as described previously (Ooga and Wakayama 2017; Ooga et al., 2018a), and observed under a confocal microscope (FV1200; Olympus Corporation, Tokyo, Japan). The mobile fraction was assessed as described in our previous study.

Immuno-staining

After zFRAP analysis and observation, the zygotes were fixed with $4 \%$ paraformaldehyde containing $0.2 \%$ Triton $\mathrm{X}-100$ for $20 \mathrm{~min}$. After washing three times with PBS containing $1 \%$ BSA and $0.2 \%$ Tween 20 , the zygotes were incubated with primary antibodies against H3K9me3 (ab8898; Abcam, Cambridge, MA, USA) diluted in PBS containing $1 \%$ BSA and $0.1 \%$ Triton X-100 at $4{ }^{\circ} \mathrm{C}$ overnight. After washing three times with PBS containing $1 \%$ BSA and $0.2 \%$ Tween 20 , the zygotes were incubated with a secondary antibody (Alexa 568 conjugated anti rabbit IgG mouse IgG). The stained zygotes were mounted on PBS containing 4',6-diamidino-2-phenylindole.

460

Statistical analysis

All statistical analyses were performed using Prism 9 software (GraphPad

463 Software, Inc., San Diego, CA, USA) with one-way analysis of variance (ANOVA) 
464 followed by Tukey's multiple comparisons test or the paired $t$-test (for parental 465 asymmetry analysis). A probability $(p)$ value of $<0.05$ was considered statistically 466 significant.

467

468 


\section{Competing interest statement}

470

The authors have no competing interests to declare.

471

472

\section{Acknowledgments}

473

We thank Drs. S. Funaya, Y. Fujimoto, Mr. K. Kazama, D. Ito, Y. Kikuchi, M.

474 Nakamura, and Miss C. Yamaguchi for critical and useful comments on the manuscript.

475 Financial support for this research was provided by a Grant-in-Aid for Young Scientists

476 (grant no. 19K16012) and Research Grant for Young Scholars funded by Yamanashi

477 Prefecture to M.O., the Asada Science Foundation and the Canon Foundation (grant no.

478 M20-0006) to T.W.

479

480 Author contributions.

481

M.O and T.W conceived and designed this study. M.O. performed most of the

482

experiments and R.I., S.W. and S.K. performed some of the experiments. M.O., R.I.,

483

S.W., S.K., and T.W. analyzed all data. M.O. and T.W. wrote the manuscript. All

484 authors read and edited the manuscript.

485

486

\section{References}

487 1. Ooga, M., Fulka, H., Hashimoto, S., Suzuki, M. G. \& Aoki, F. Analysis of

after photobleaching. Epigenetics 11, (2016). 
490

491

492

493

494

495

496

497

498

499

500

501

502

503

504

505
2. Adenot, P. G., Mercier, Y., Renard, J. P. \& Thompson, E. M. Differential H4 acetylation of paternal and maternal chromatin precedes DNA replication and differential transcriptional activity in pronuclei of 1-cell mouse embryos.

Development 124, 4615-4625 (1997).

3. Aoki, F., Worrad, D. M. \& Schultz, R. M. Regulation of transcriptional activity during the first and second cell cycles in the preimplantation mouse embryo. Dev.

Biol. 181, 296-307 (1997).

4. Burton, A. \& Torres-Padilla, M. E. Epigenetic reprogramming and development:

A unique heterochromatin organization in the preimplantation mouse embryo.

Brief. Funct. Genomics 9, 444-454 (2010).

5. Liu, W. et al. Asymmetric Reprogramming Capacity of Parental Pronuclei in Mouse Zygotes. Cell Rep. 6, 1008-1016 (2014).

6. Kishigami, S., Wakayama, S., Nguyen, V. T. \& Wakayama, T. Similar time restriction for intracytoplasmic sperm injection and round spermatid injection into activated oocytes for efficient offspring production. Biol. Reprod. 70, 18639 (2004). 
506 7. Brykczynska, U. et al. Repressive and active histone methylation mark distinct

507 promoters in human and mouse spermatozoa. Nat. Struct. Mol. Biol. 17, 679-687

$508 \quad(2010)$

509 8. Hammoud, S. S. et al. Distinctive chromatin in human sperm packages genes for

$510 \quad$ embryo development. Nature 460, 473-478 (2009).

511 9. Paradowska, A. S. et al. Genome wide identification of promoter binding sites

512 for H4K12ac in human sperm and its relevance for early embryonic

513 development. Epigenetics 7, 1057-1070 (2012).

514 10. Yamaguchi, K. et al. Re-evaluating the Localization of Sperm-Retained Histones

515 Revealed the Modification-Dependent Accumulation in Specific Genome

$516 \quad$ Regions. Cell Rep. 23, 3920-3932 (2018).

517 11. Sharma, U. et al. Biogenesis and function of tRNA fragments during sperm

518 maturation and fertilization in mammals. Science (80-. ). 351, 391-396 (2016).

519 12. Trigg, N. A., Eamens, A. L. \& Nixon, B. The contribution of epididymosomes to

520 the sperm small RNA profile. Reproduction vol. 157 R209-R223 (2019).

521 13. Teperek, M. et al. Sperm is epigenetically programmed to regulate gene 
transcription in embryos. Genome Res. 26, 1034-1046 (2016).

523

14. Chen, X. et al. Human sperm tsRNA as potential biomarker and therapy target

524 for male fertility. Reproduction 161, 111-122 (2021).

525

15. Ooga, M. et al. Chd9 mediates highly loosened chromatin structure in growing mouse oocytes. Biochem. Biophys. Res. Commun. 500, 583-588 (2018).

16. Funaya, S., Ooga, M., Suzuki, M. G. \& Aoki, F. Linker histone H1FOO regulates the chromatin structure in mouse zygotes. FEBS Lett. 592, (2018).

529

17. Sharma, U. Paternal contributions to offspring health: Role of sperm small rnas Biol. 7, 1-15 (2019).

532

18. Yan, W. et al. Birth of mice after intracytoplasmic injection of single purified sperm nuclei and detection of messenger RNAs and microRNAs in the sperm nuclei. Biol. Reprod. 78, 896-902 (2008).

19. Schuster, A. et al. SpermBase: A Database for Sperm-Borne RNA Contents. Biol. Reprod. 95, 99-99 (2016).

537 20. Rathke, C., Baarends, W. M., Awe, S. \& Renkawitz-Pohl, R. Chromatin 

1839, 155-168 (2014).

21. Inoue, A. \& Zhang, Y. Nucleosome assembly is required for nuclear pore complex assembly in mouse zygotes. Nat. Struct. Mol. Biol. 21, 609-616 (2014).

22. Ooga, M. et al. Changes in H3K79 methylation during preimplantation development in mice. Biol. Reprod. 78, (2008).

23. Egli, D., Birkhoff, G. \& Eggan, K. Mediators of reprogramming: Transcription factors and transitions through mitosis. Nat. Rev. Mol. Cell Biol. 9, 505-516

24. Kishigami, S., Van Thuan, N., Wakayama, S., Hikichi, T. \& Wakayama, T. A novel method for isolating spermatid nuclei from cytoplasm prior to ROSI in the mouse. Zygote 12, 321-327 (2004).

25. Bui, H. T. et al. Essential role of paternal chromatin in the regulation of 141, 67-77 (2011). 

(2018).

27. Quinn, P. \& Begley, A. J. Effect of human seminal plasma and mouse accessory (1984).

28. Chatot, C. L., Lewis, L. J., Torres, I. \& Ziomek, C. A. Development of 1-cell gland extracts on mouse fertilization in vitro. Aust. J. Biol. Sci. 37, 147-152

29. Wakayama, S., Kishigami, S. \& Wakayama, T. Improvement of mouse cloning embryos from different strains of mice in CZB medium. Biol. Reprod. 42, 432440 (1990).

30. Konno, S. et al. Removal of remodeling/reprogramming factors from oocytes and the impact on the full-term development of cloned embryos. Development 147, (2020). 
570 32. Ooga, M. \& Wakayama, T. FRAP analysis of chromatin looseness in mouse zygotes that allows full-Term development. PLoS One 12, (2017).

572 33. Ooga, M., Funaya, S., Aoki, F. \& Wakayama, T. Zygotic fluorescence recovery

573 after photo-bleaching analysis for chromatin looseness that allows full-term

574 development. J. Vis. Exp., (2018).

575

576

577 


\section{Supplemental Fig. 1}

A
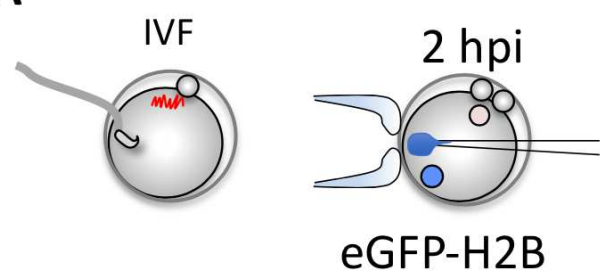

mRNA injection

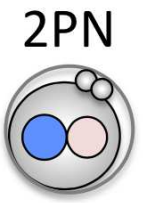

B ำ 9

C

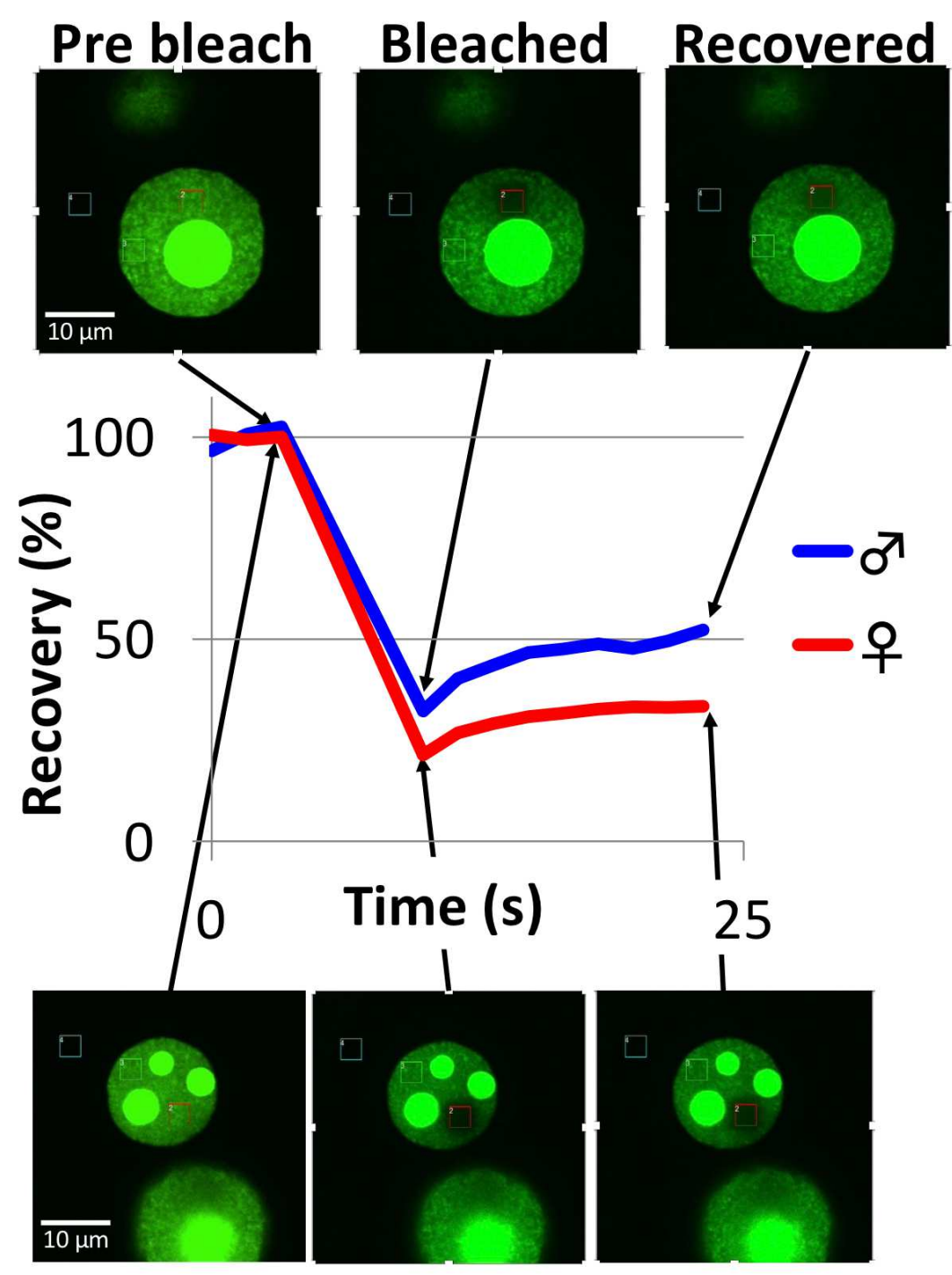

579

580 Supplemental Fig. 1 
582 (A) In our previous study, IVF-derived zygotes were microinjected with mRNA at 2 hpi.

583 (B) At 8 hpi, parental PNs were easily distinguished by size, as a male PN is larger than 584 a female PN. Sufficient expression of eGFP-H2B was confirmed. (C) For zFRAP analysis, 585 a specific region of interest (ROI) in the PNs was selected and bleached. Red rectangle 586 indicates bleached ROI, green is reference, and light blue is background. Compared to 587 pre-bleaching, the fluorescence level drastically decreased after bleaching and then 588 gradually recovered. Male PNs always exhibited greater fluorescence than the female 589 PNs. Fluorescence levels at several points were plotted as a "recovery curve." 


\section{Supplemental Fig. 2}
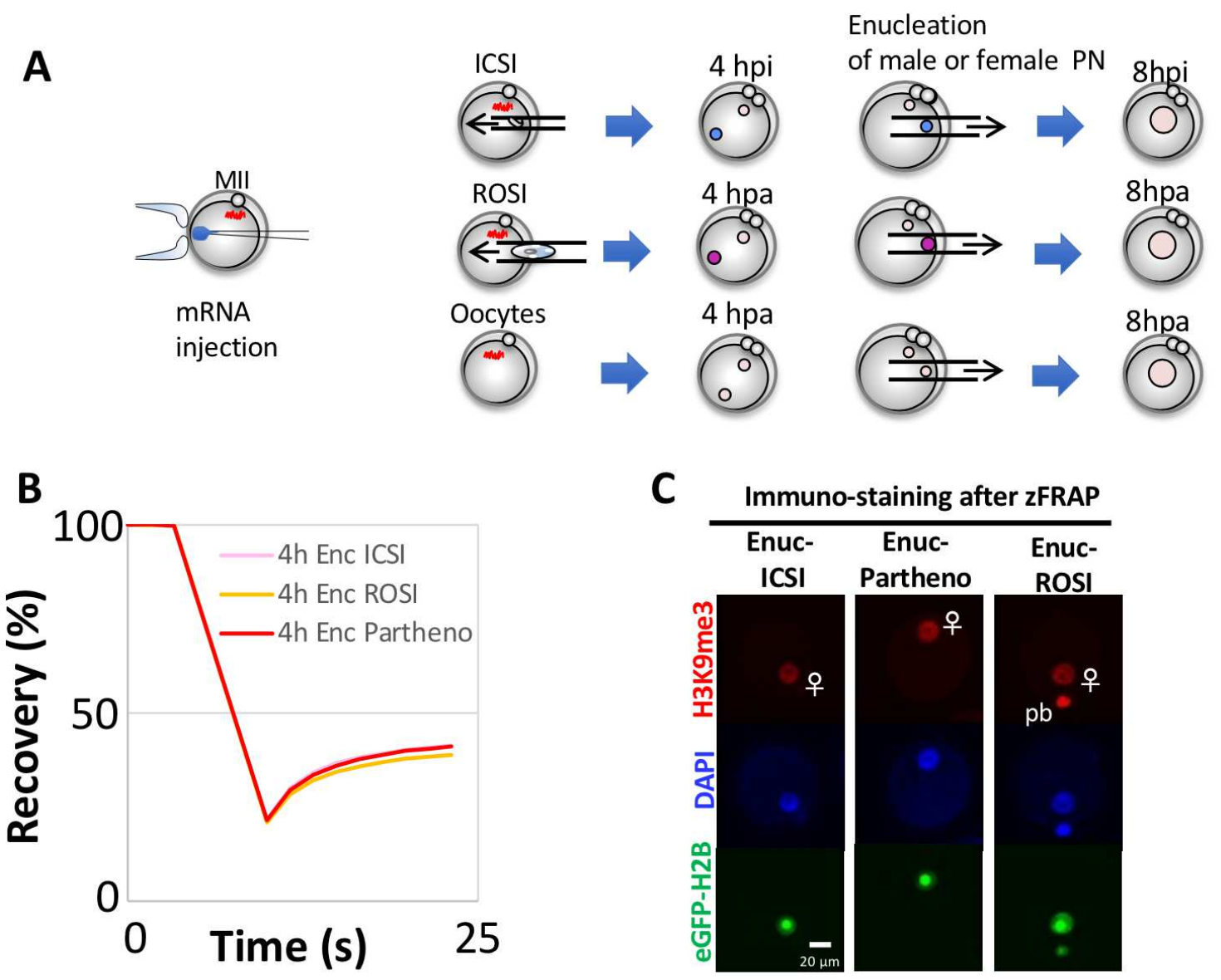

591

592 Supplemental Fig. 2

593 Dependency of chromatin compaction of fPN on sp-mPN

594 (A) Illustration of the preparation of mPN-enucleated partheno-zygotes at 4 hpi (B)

595 Recovery curve of mPN-enucleated partheno-zygotes. (C) In order to confirm correct PN

596 selection, after zFRAP analysis, the zygotes were subjected immuno-staining of

597 H3K9me3, as a fPN marker.

598

599

600

601 


\section{Supplemental Fig. 3}

602

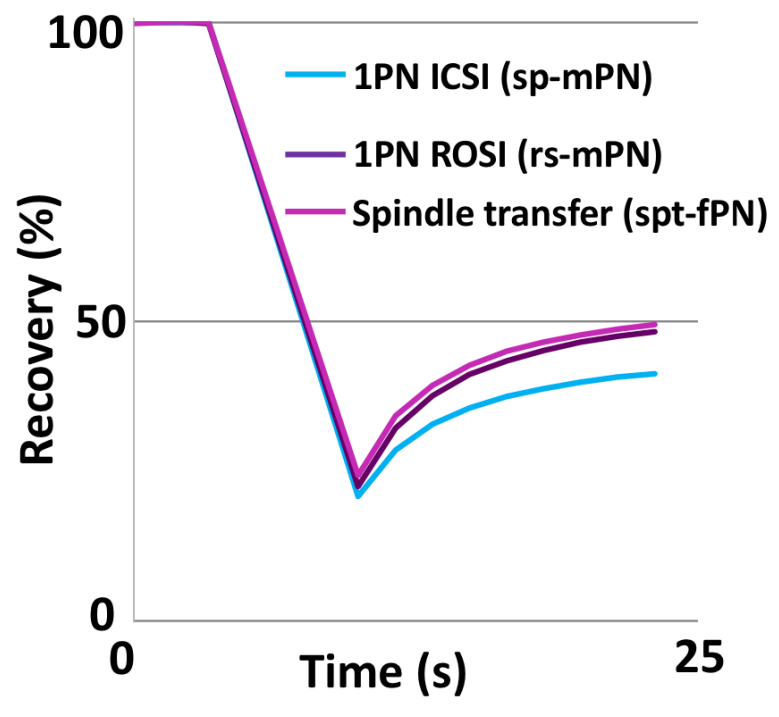

603 Supplemental Fig. 3

604 Sperm-derived chromatin compaction ability functions to sp-mPN itself.

605 Recovery curve of 1PN-zygotes. 1PN ICSI have only sp-mPN (sperm-derived mPN),

606 1PN ROSI have only rs-mPN (round spermatid-derived mPN), and spindle transfer have 607 only spt-fPN (spindle transfer-derived fPN).

608

609 
Supplemental Fig. 4

A
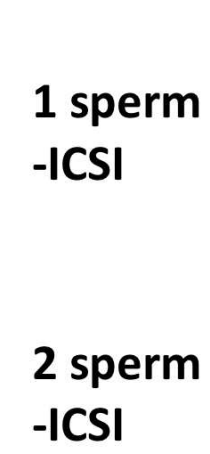

enucleated
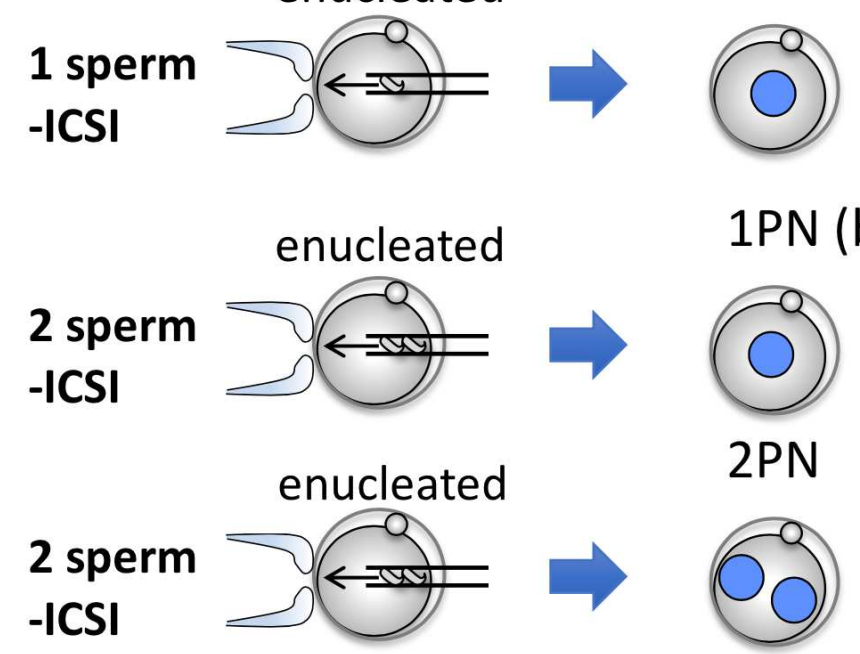

"1ठ (1sp)"

1PN (by PN fusion)

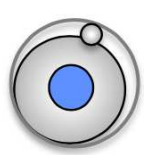

"1ठ (2sp)"

2PN

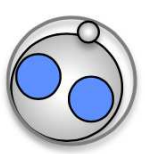

"2 ठ" (2sp)"

3PN
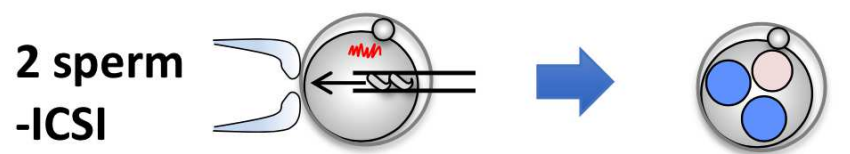

"2ळ +1 우 (2sp)"

\section{B}

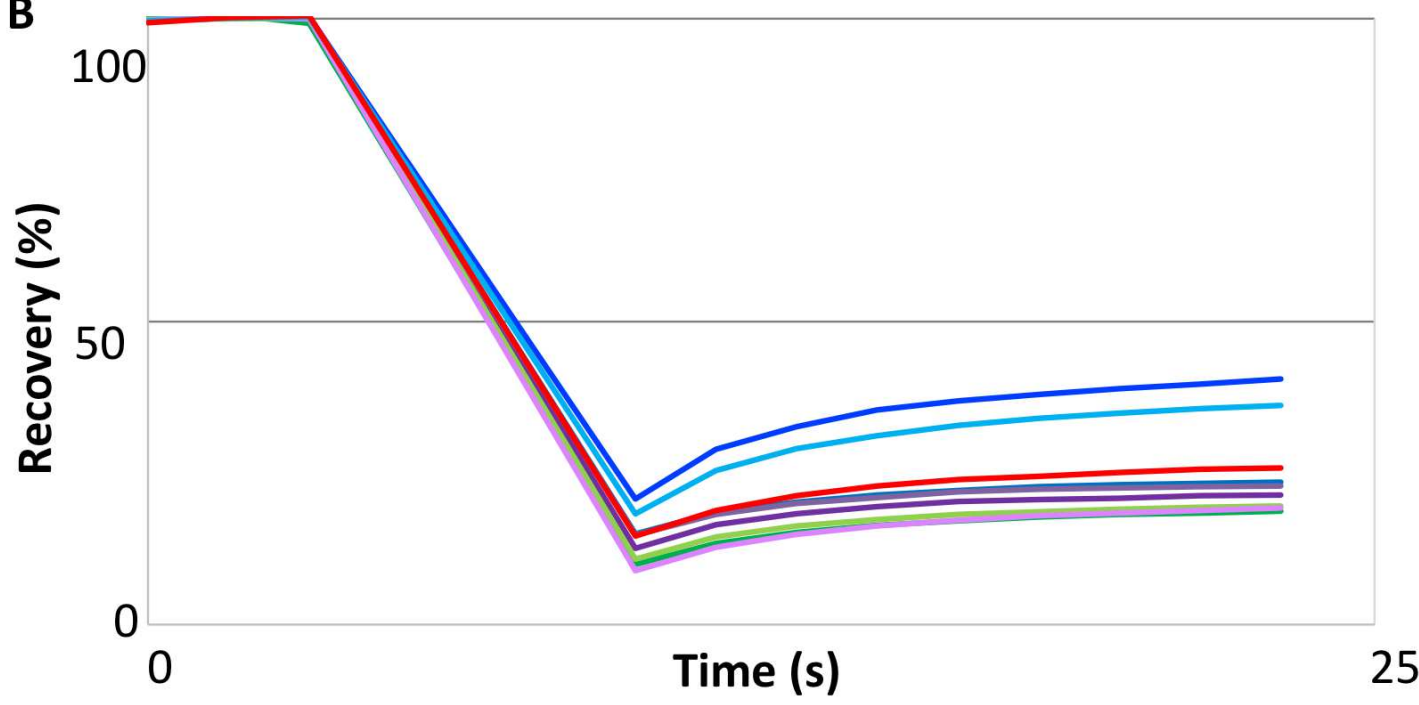

610

$$
\begin{aligned}
& -1 \sigma^{\top}(1 \mathrm{sp}) \quad-1 \sigma^{\top}(2 \mathrm{sp}) \quad-2 \sigma^{\top}(2 \mathrm{sp}) \\
& -2 \sigma^{\top}(2 \mathrm{sp}) \quad-2 \sigma^{\top}+1 \text { 우 male }-2 \sigma^{\top}+1 \text { 우 male } \\
& -2 \sigma^{\top}+1 \text { 우 female -Ctrl ICSI Male -Ctrl ICSI Fem }
\end{aligned}
$$

611 Supplemental Fig. 4

612 Chromatin compaction is dependent on the number of microinjected sperm 
613 (A) Schematic illustration of the preparation of zygotes of " $10^{\prime}$ (1sp)," "1 $10^{\prime}$ (2sp)," " $20^{\prime \prime}$

614 (2sp)" and " $20^{\prime}+1$ ( $(2 \mathrm{sp})$." One or two sperm were microinjected into enucleated or 615 un-enucleated MII oocytes. "1ळ (1sp)" indicates that one sperm was injected, which 616 resulted in the formation of a single sp-mPN. (B) Recovery curves of ICSI-zygotes 617 microinjected with one or two sperm.

618

619 


\section{Supplemental Fig. 5}

A

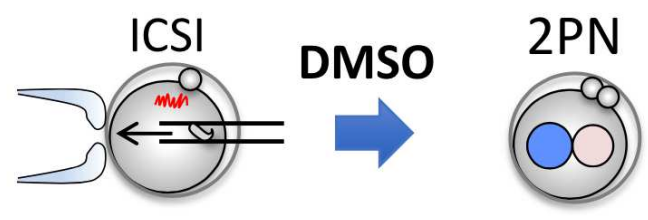

$1 \%$ DMSO
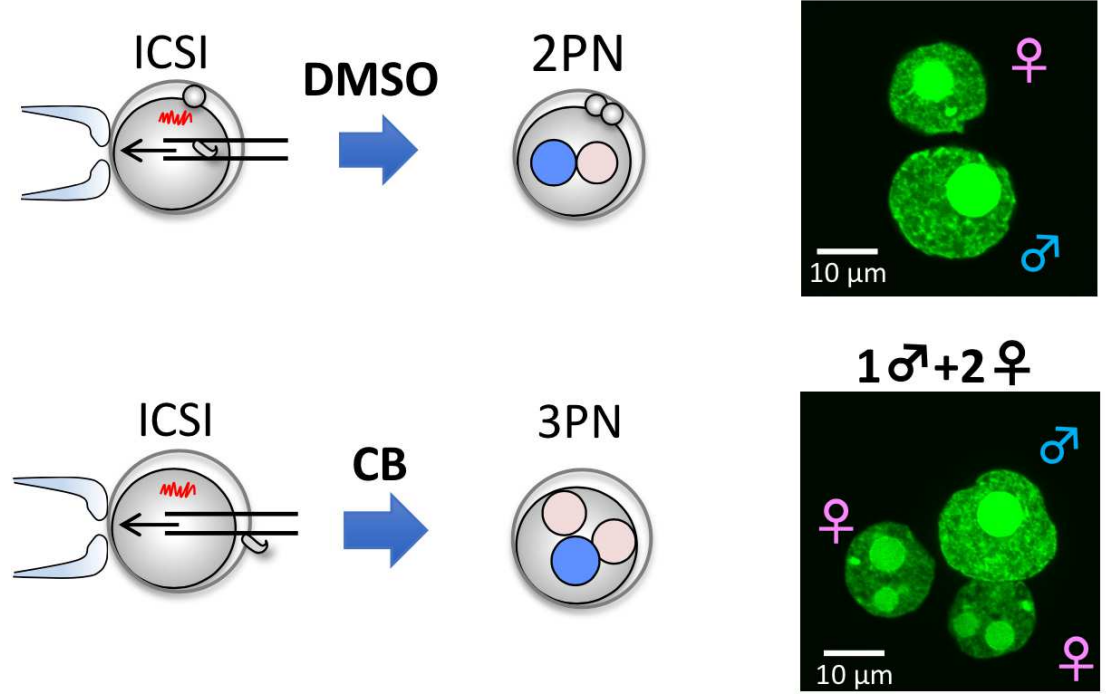

\section{B}

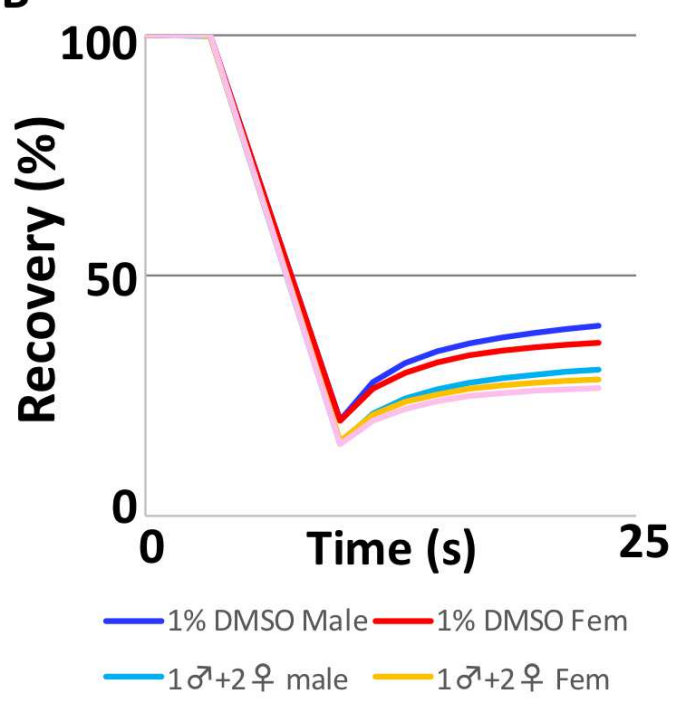

620

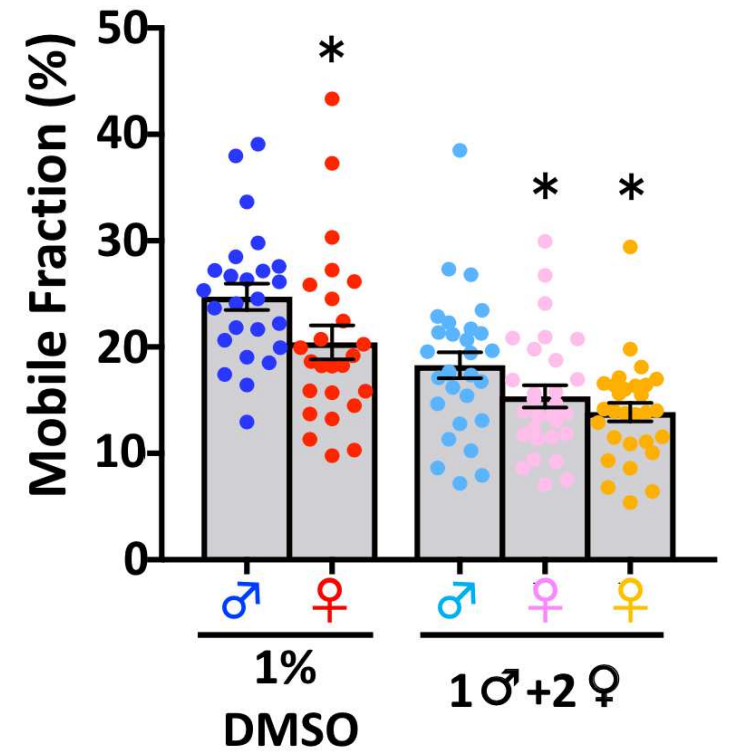

\section{Supplemental Fig. 5}

622 Increases of fPN did not disrupt parental asymmetric pattern.

623 (A) Schematic illustration of the preparation of zygotes of " $10^{\star}+29 . " \mathrm{CB}$ is an inhibitor

624 of cytokinesis, which increases the fPN in the treated zygotes. Fluorescence images of 625 the zygotes are shown. Two fPNs are shown. (B) Recovery curve and average MF scores 
626 of " $10 " 2 q$ " and control zygotes are shown. Blue, red, light blue, pink, and orange dots 627 are MF scores of sp-mPN, fPN in control zygotes, and sp-mPN and two fPNs in CB 628 treated-zygotes, respectively.

629

630 


\section{Supplemental Fig. 6}

A
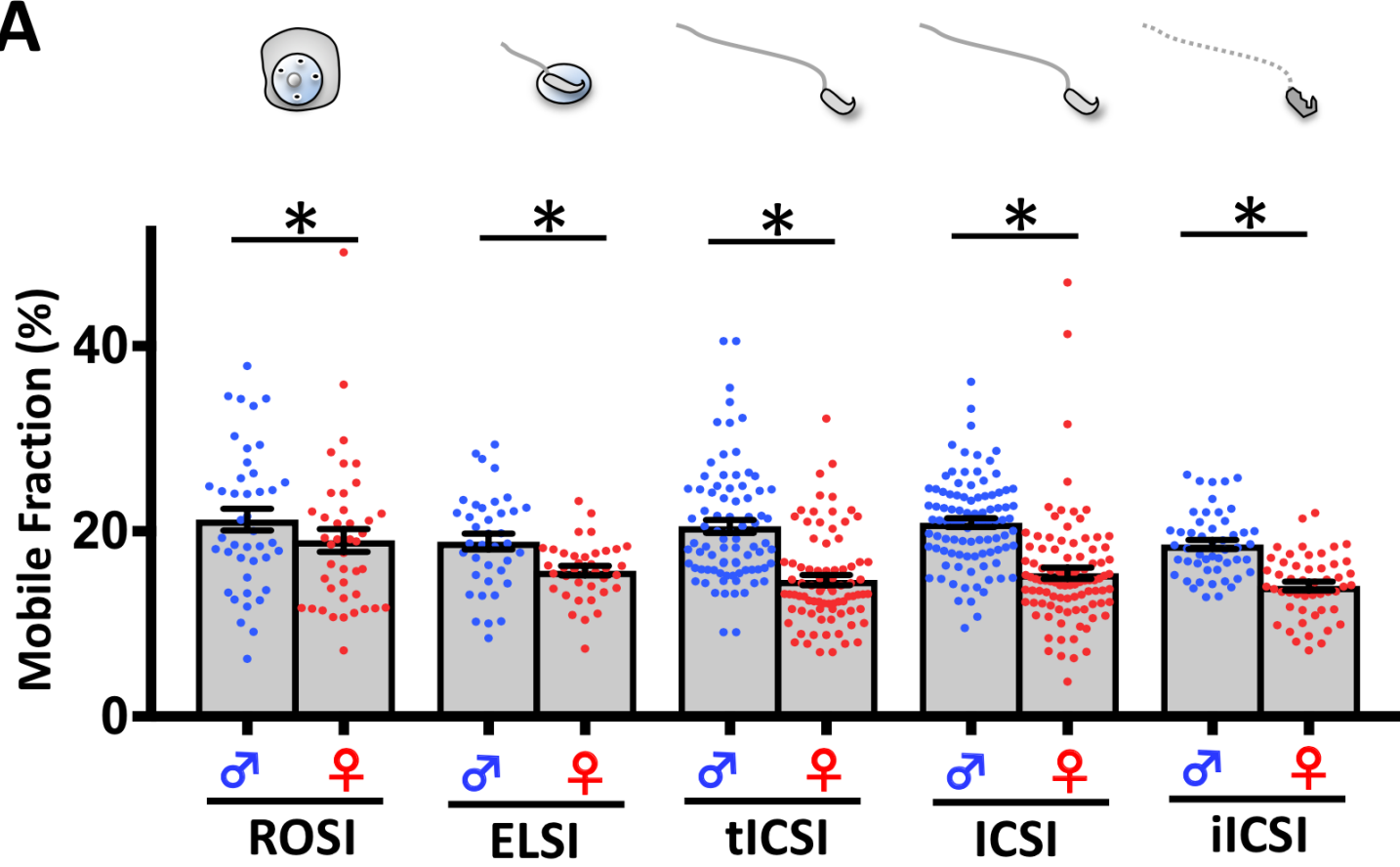

B

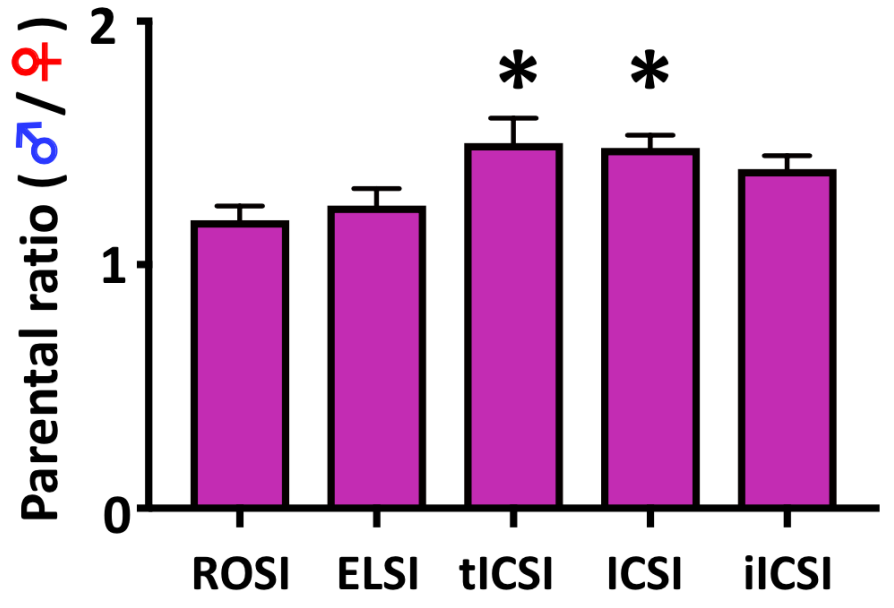

632 Supplemental Fig. 6

633 Male germ cells acquired the ability to compact chromatin and resistance during 634 spermiogenesis

635 (A) zFRAP analysis with ROSI, ELSI, tICSI, ICSI, and ICSI with inactivated sperm 636 (iICSI) was performed. The average MF scores of the zygotes are shown. Blue and red 
637 dots are the MF scores of the $\mathrm{mPN}$ and fPN, respectively. (B) Bar graph of the average $638 \mathrm{MF}$ score of the $\mathrm{mPN} / \mathrm{fPN}$ ratio. The asterisks indicate significant differences as 639 compared with ROSI by one-way ANOVA followed by Tukey's multiple comparisons 640 test.

641

642 


\section{Supplemental Fig. 7}

A

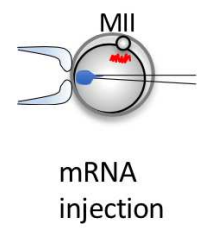

B

643

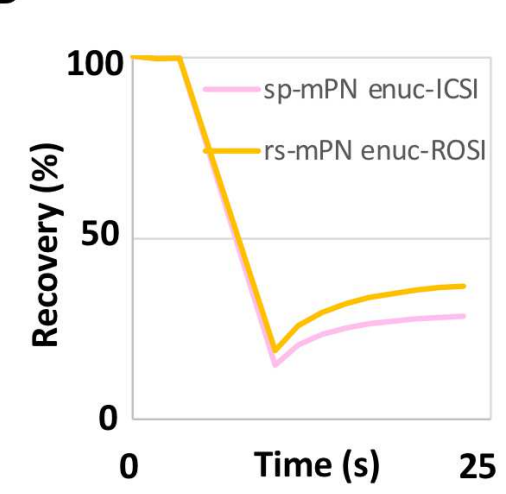

C

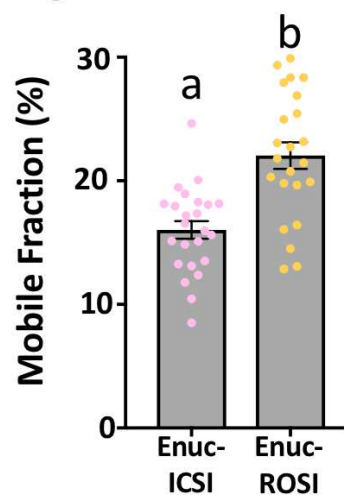

Enucleation

of male PN

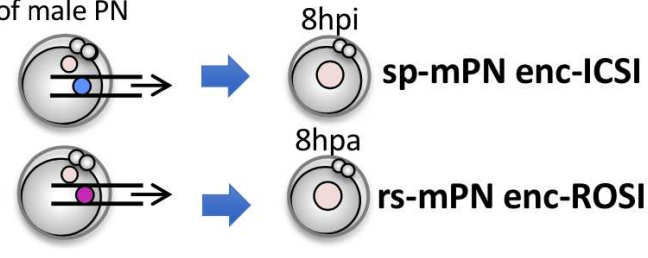

D Immuno-staining

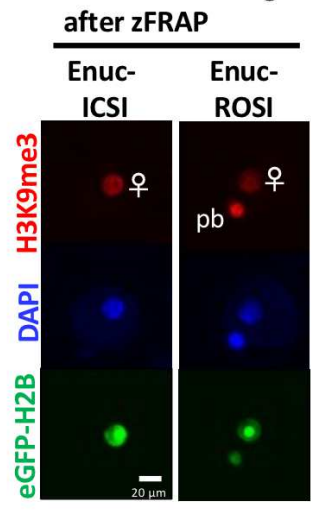

\section{Supplemental Fig. 7}

645 Round spermatid did not harbor chromatin compaction ability

646 (A) Schematic illustration of the preparation of ICSI- and ROSI-zygotes without mPN.

647 (B) Recovery curve of mPN-enucleated ICSI-(sp-mPN enuc-ICSI) and ROSI(rs-mPN 648 enuc-ROSI)-zygotes. (C) The average MF scores are shown. Pink and orange dots 649 indicate the MF score of each fPN of the zygotes. (D) Immuno-staining of H3K9me3 in 650 zygotes after zFRAP analysis. H3K9me3 signals indicating correct discrimination of 651 parental PN during the enucleation process.

652 


\section{Supplemental Fig. 8}

A

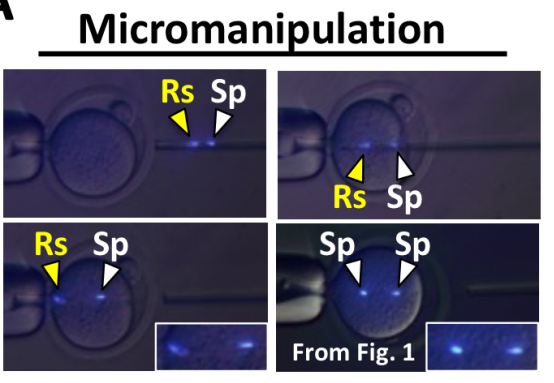

C

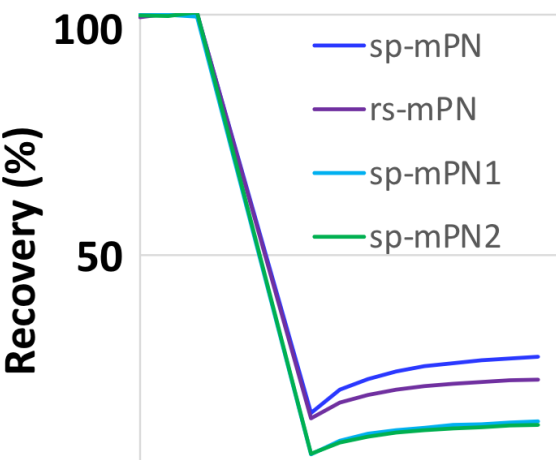

0

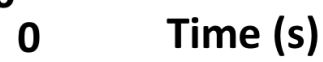

25

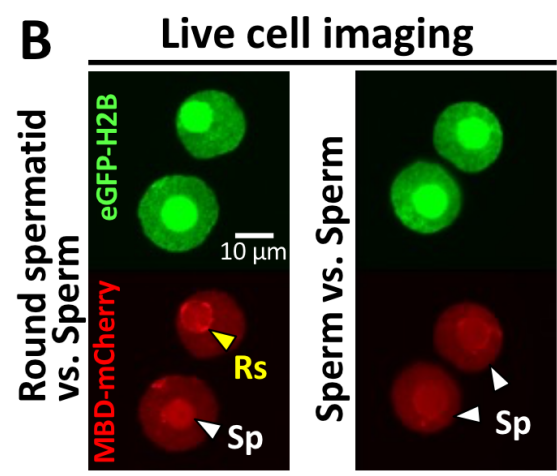

D

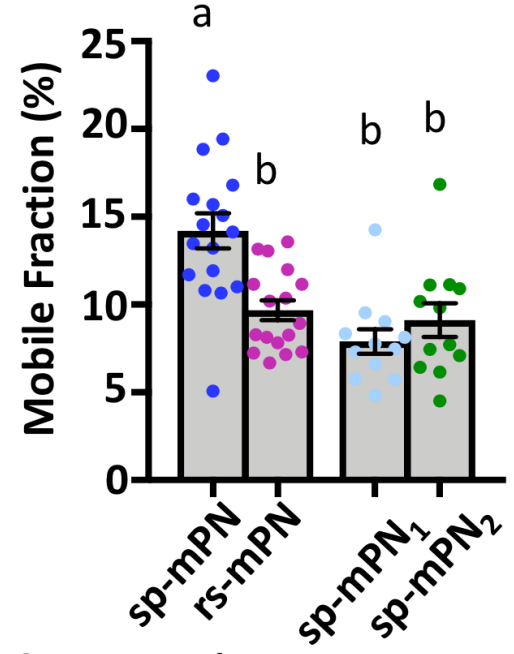

\section{E}

Immuno-staining (after zFRAP)
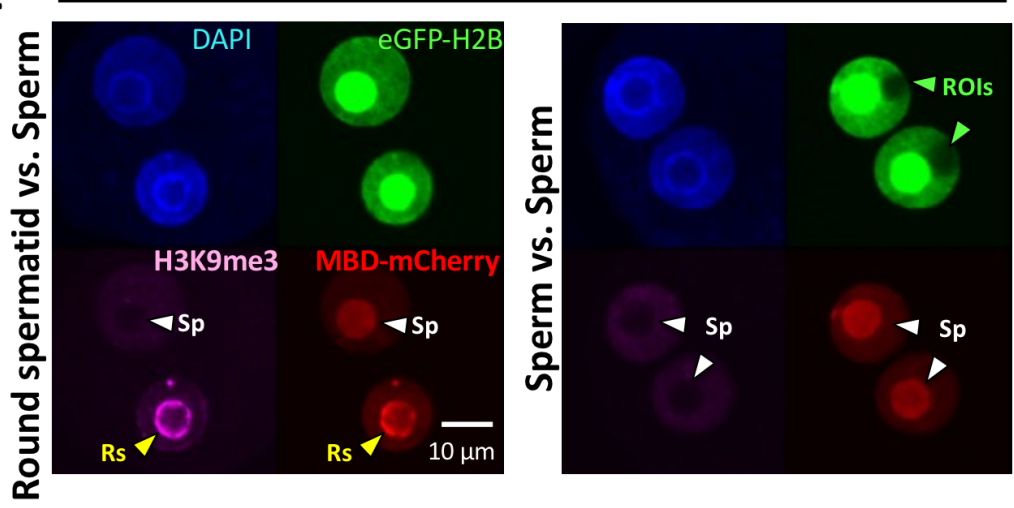

656

\section{Supplemental Fig. 8}

658 Round spermatids were not resistant to the chromatin compaction effect of sperm

659 (A) "Sperm head and round spermatid" or "sperm head and sperm head" were co-injected 660 into the enucleated oocytes at the same time. Yellow "Rs" and white "Sp" indicate round 
661 spermatid and sperm, respectively. The insets are images at higher magnification. DNA

662 was stained with Hoechst. (B) MBD-mCherry expression in the live zygotes during 663 zFRAP analysis. Upper panel is eGFP-H2B expression. Lower red panel indicates MBD664 mCherry expression. Triangle indicates the perinucleolar ring where MBD-mCherry was 665 enriched in male PNs derived from round spermatid but not sperm. (C) Recovery curve 666 indicating the average fluorescence recovery rate. sp-mPN, rs-mPN, sp-mPN\#1, and sp$667 \mathrm{mPN \# 2}$ indicate the score of male PN derived from round spermatid and spermatozoa, 668 and co-injected spermatozoon, respectively. (D) MF scores of 2PN androgenic zygotes 669 are shown. Gray bar graph of average MF values. Single dots indicate the mobile fraction 670 score obtained from either mPN derived from round spermatid or sperm. Different 671 characters indicate significant differences ( $p<0.05$, by one-way ANOVA and Tukey’s 672 multi comparisons test). Error bar indicates the SE.

673 (E) Immunocytochemial analysis with antibody against H3K9me3. To confirm 674 discrimination of the derivation of male PNs using MBD-mCherry, since the marker for 675 male PN derived from round spermatid, H3K9me3, was stained. Purple indicates immno676 staining of H3K9me3, which only male PNs derived from round spermatid showed. 677 Green triangle indicates the ROIs that were bleached during zFRAP analysis.

678

679

680

681

682

683

684 


\section{Supplemental Fig. 9}

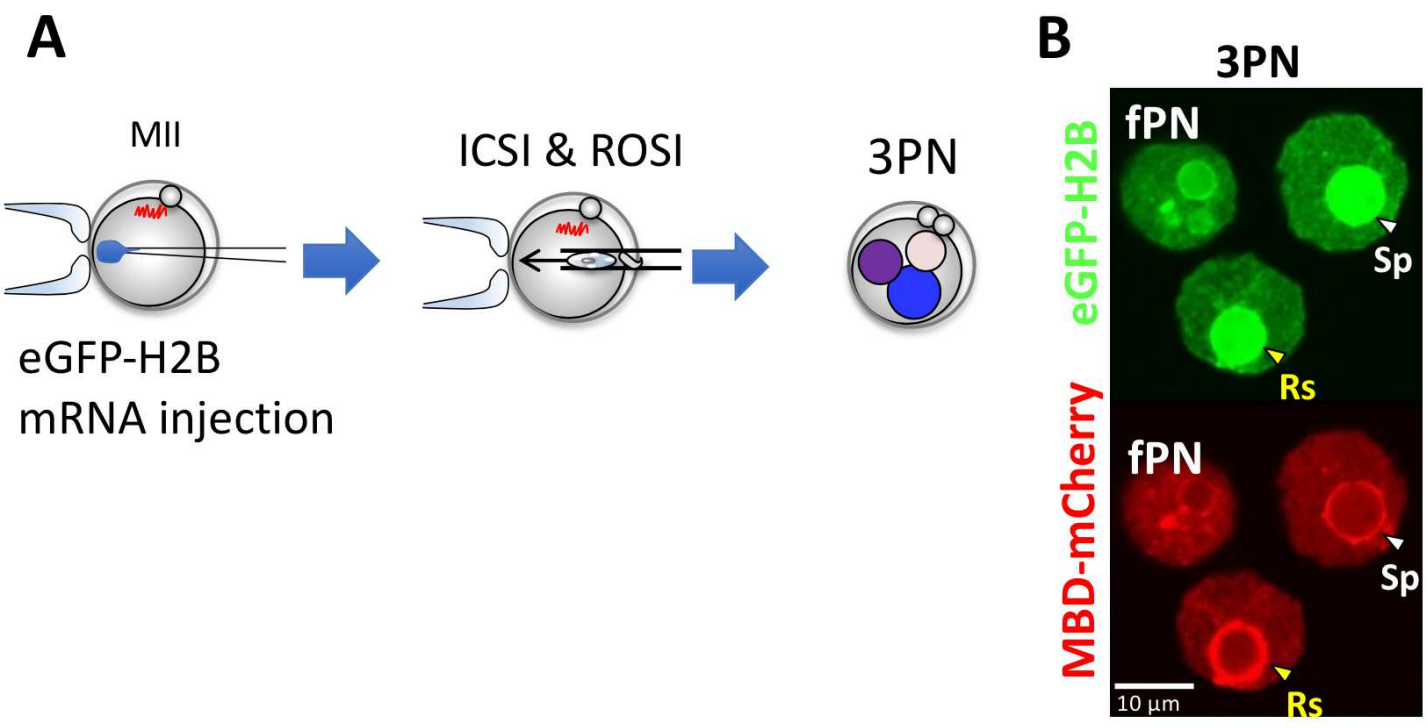

\section{C}

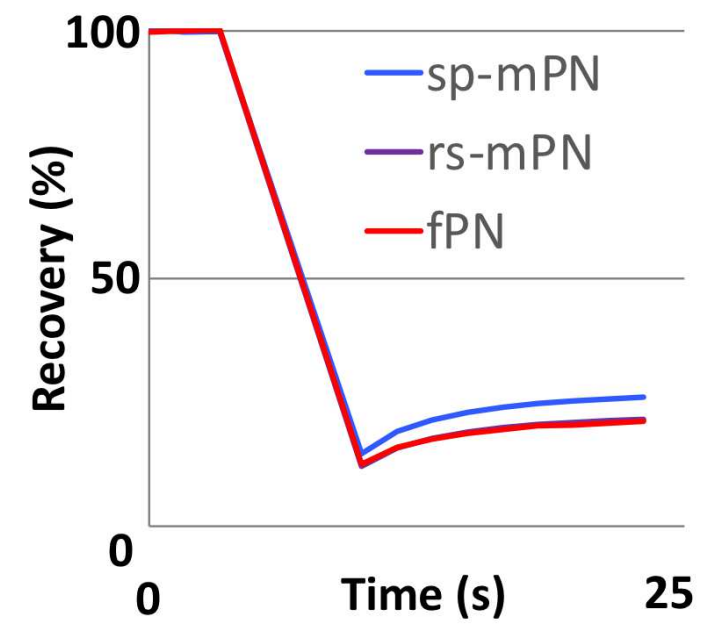

685

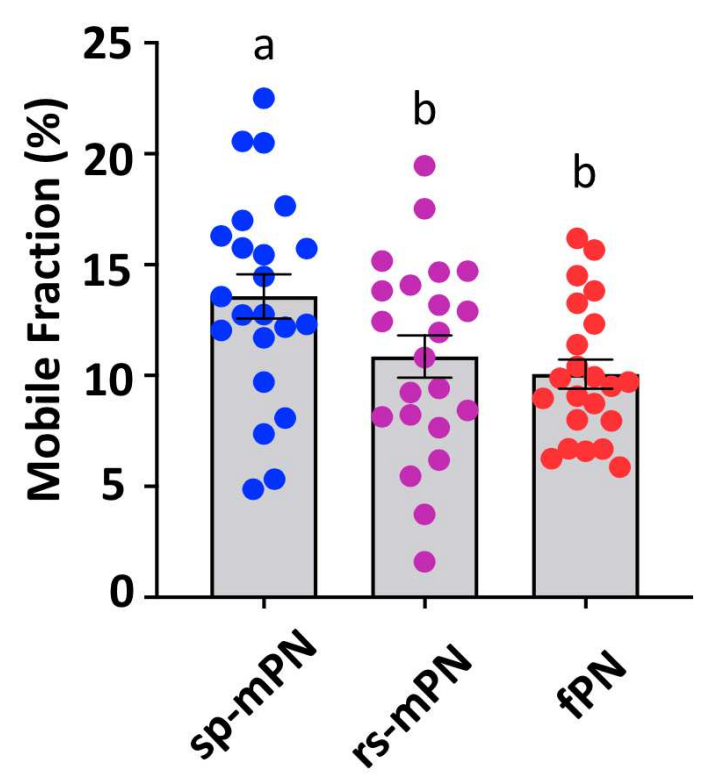

686 Supplemental Fig. 9

687 Chromatin derived from round spermatid could be condensed to the same level as $f P N$

688 (A) Schematic illustration of the preparation of zygotes with sp-mPN, rs-mPN, and fPN.

689 Sperm and round spermatids were co-injected into un-enucleated MII oocytes. (B)

690 Fluorescence image of zygotes harboring the 3PN. MBD-mCherry showed preferential 
691 localization to the nucleolar ring of rs-mPN to distinguish the derivation of mPNs. (C),

692 (D) Recovery curve and MF scores of sp-mPN, rs-mPN, and fPN in 3PN-zygotes.

693

694 
Supplemental Fig. 10

695

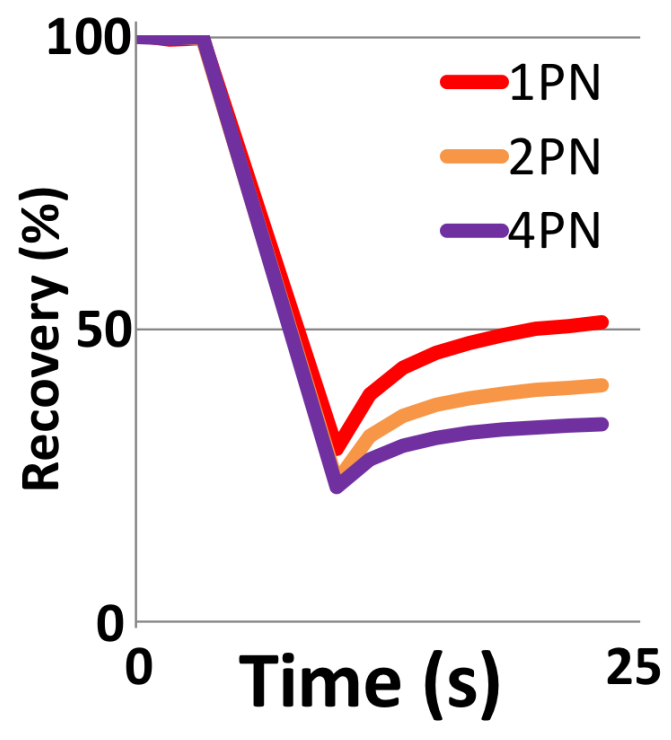

696 Supplemental Fig. 10

697 Recovery curve of 1, 2, and 4PN parthenogenetic zygotes.

698 


\section{Supplemental Fig. 11}

A

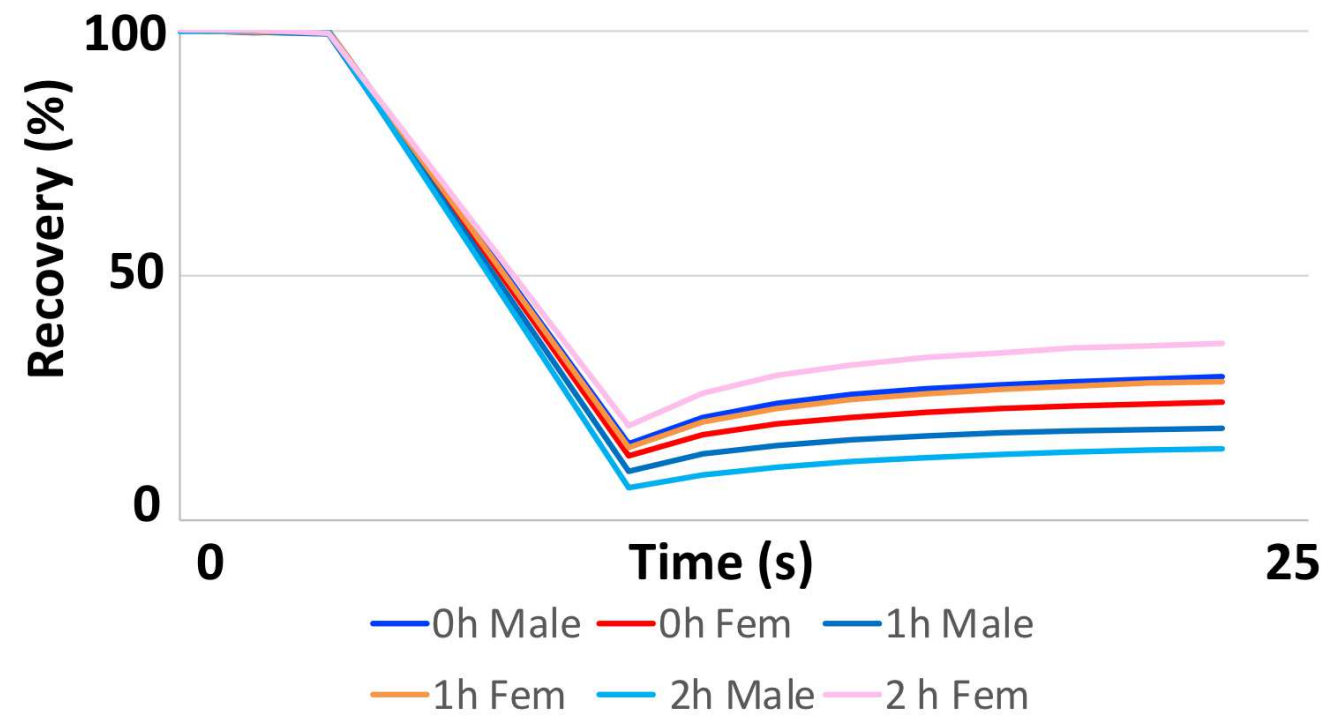

B
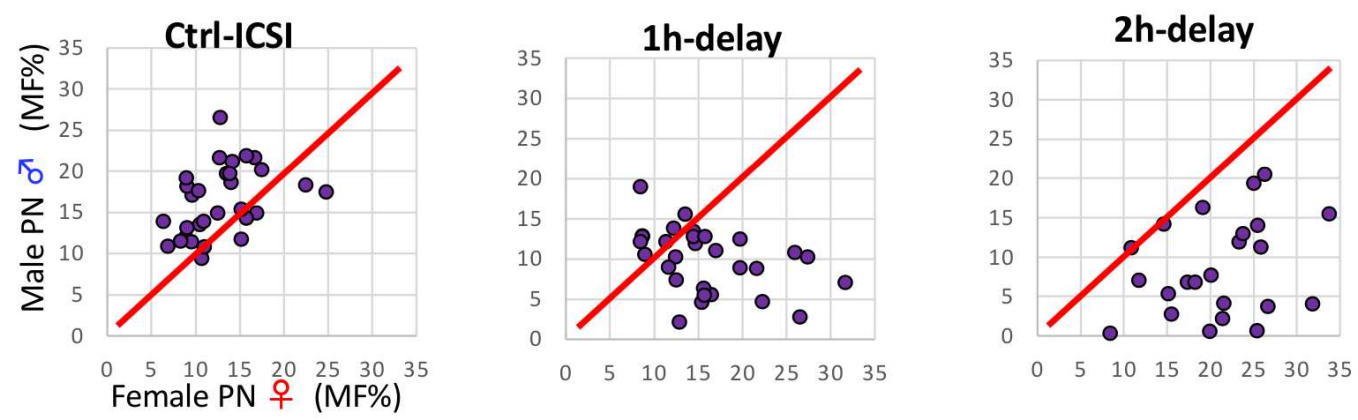

699

Supplemental Fig. 11

701 (A) Recovery curve of control and 1, 2 h-delay ICSI zygotes.

702

(B) Purple single dots indicate MF scores of parental individual pronuclei. Red line

703 indicates the $\sigma^{x}=$ Q border line. Left side: $\sigma^{x}>$ Q , right side: $\sigma^{x}<$ ․

704

705

706

707 
Supplemental Fig. 12

708
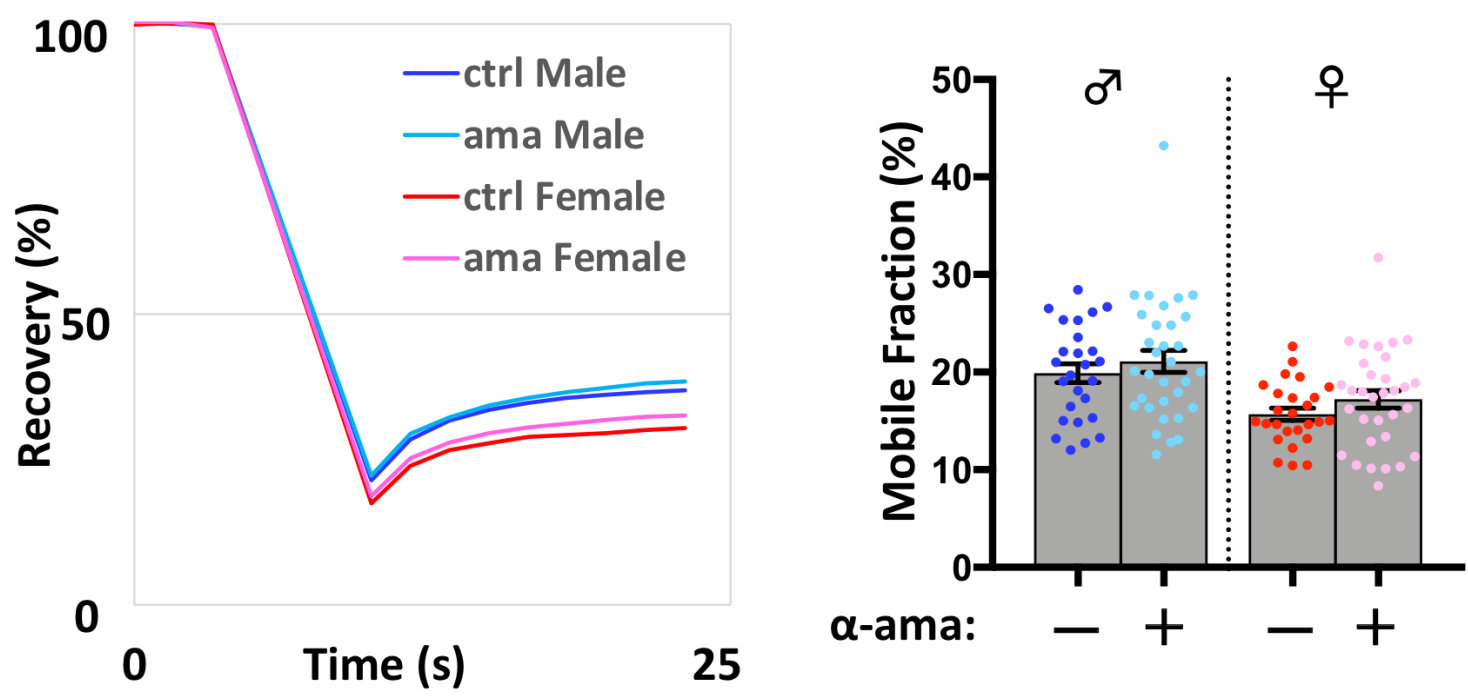

709 Supplemental Fig. 12

710 (A) Recovery curve of control and alpha-amanitin (RNA pol II inhibitor, $\alpha$-ama) treated

711 IVF-zygotes. Ctrl indicates non-treated zygotes. (B) The MF scores of the zygotes are 712 shown in the bar graph.

713

714 


\section{Supplemental Fig. 13}

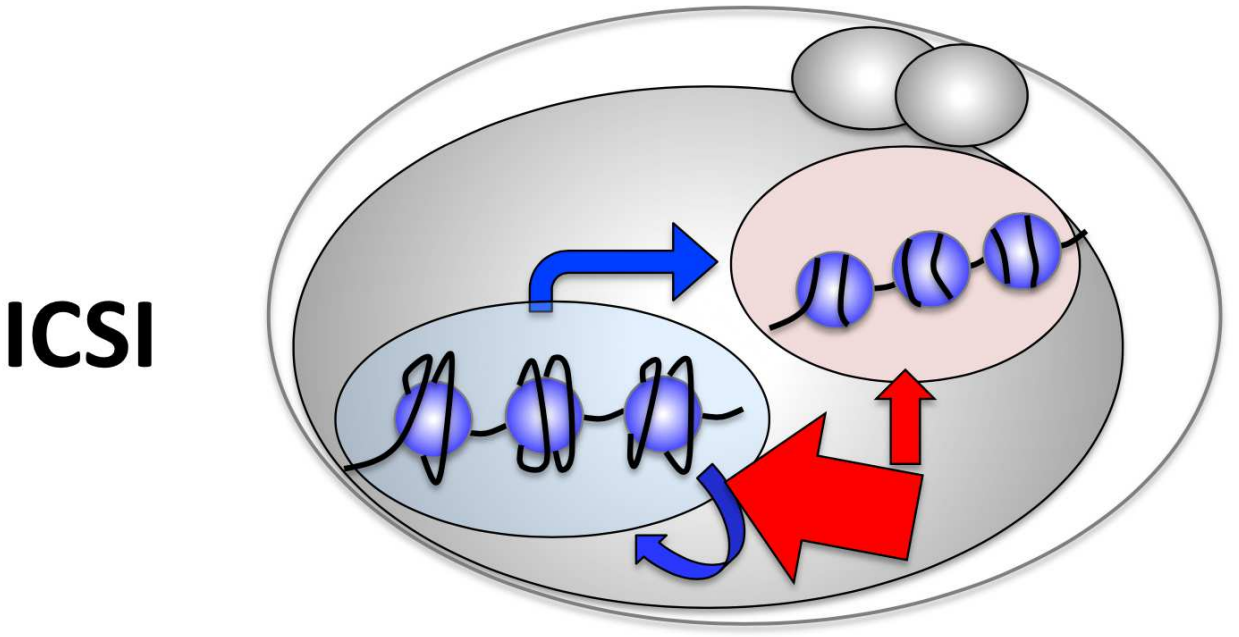

\section{Chromatin Compaction (sperm)}

\section{Chromatin Relaxation (oocytes)}

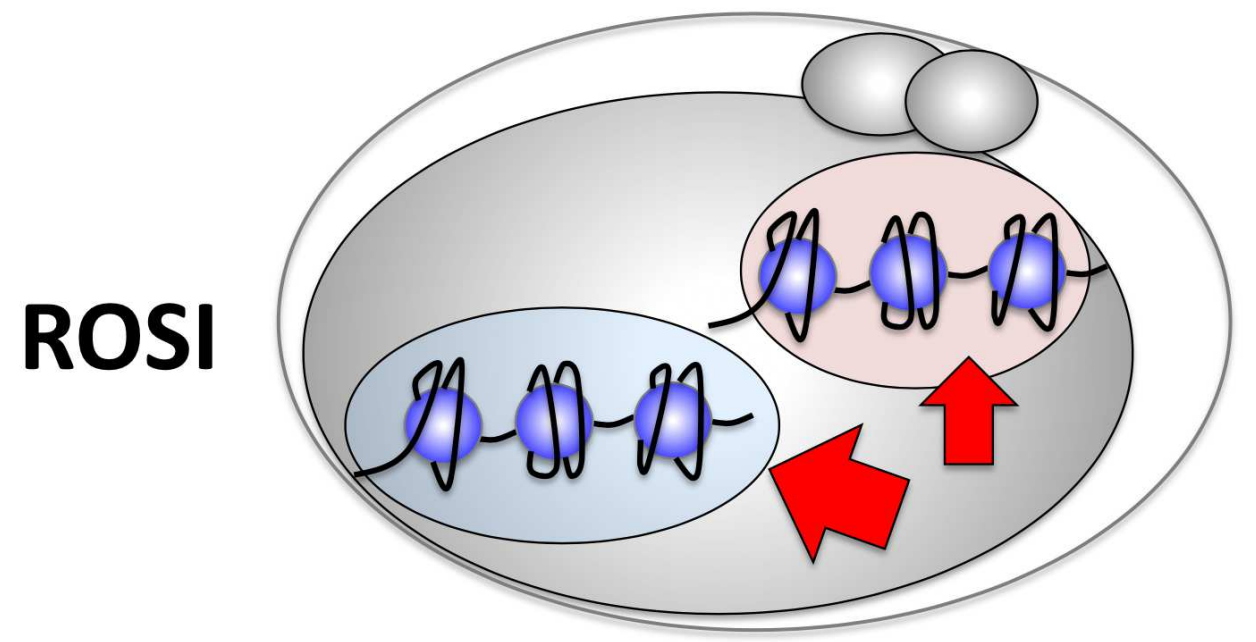

716 Supplemental Fig. 13

717 Schematic illustration of the hypothetical model in this study. In ROSI-zygotes, since

718 round spermatids lack the ability to compact chromatin, fPN could avoid chromatin

719 compaction. However, as described in the Discussion section, an rs-mPN may be able to 
720 incorporate more maternal factors (including chromatin relaxer) from the cytoplasm,

721 resulting in a slightly more relaxed chromatin structure in an rs-mPN than a fPN.

722

723

724

725

726 